\title{
Continuous functors as a model for the equivariant stable homotopy category
}

\author{
ANDREW J BLUMBERG
}

It is a classical observation that a based continuous functor $X$ from the category of finite CW-complexes to the category of based spaces that takes homotopy pushouts to homotopy pullbacks "represents" a homology theory-the collection of spaces $\left\{X\left(S^{n}\right)\right\}$ obtained by evaluating $X$ on spheres yields an $\Omega$-prespectrum. Such functors are sometimes referred to as linear or excisive. The main theorem of this paper provides an equivariant analogue of this result. We show that a based continuous functor from finite $G-\mathrm{CW}$-complexes to based $G$-spaces represents a genuine equivariant homology theory if and only if it takes $G$-homotopy pushouts to $G$-homotopy pullbacks and satisfies an additional condition requiring compatibility with Atiyah duality for orbit spaces $G / H$.

Our motivation for this work is the development of a recognition principle for equivariant infinite loop spaces. In order to make the connection to infinite loop space theory precise, we reinterpret the main theorem as providing a fibrancy condition in an appropriate model category of spectra. Specifically, we situate this result in the context of the study of equivariant diagram spectra indexed on the category $\mathfrak{S}_{G}$ of based $G$-spaces homeomorphic to finite $G-\mathrm{CW}$-complexes for a compact Lie group $G$. Using the machinery of Mandell-May-Schwede-Shipley, we show that there is a stable model structure on this category of diagram spectra which admits a monoidal Quillen equivalence to the category of orthogonal $G$-spectra. We construct a second "absolute" stable model structure which is Quillen equivalent to the stable model structure. There is a model-theoretic identification of the fibrant continuous functors in the absolute stable model structure as functors $Z$ such that for $A \in \mathfrak{S}_{G}$ the collection $\left\{Z\left(A \wedge S^{W}\right)\right\}$ forms an $\Omega-G$-prespectrum as $W$ varies over the universe $U$. Thus, our main result provides a concrete identification of the fibrant objects in the absolute stable model structure.

This description of fibrant objects in the absolute stable model structure makes it clear that in the equivariant setting we cannot hope for a comparison between the category of equivariant continuous functors and equivariant $\Gamma$-spaces, except when $G$ is finite. We provide an explicit analysis of the failure of the category of equivariant $\Gamma$-spaces to model connective $G$-spectra, even for $G=S^{1}$.

55P42; 55P47, 55P91 


\section{Introduction}

One of the striking successes in the development of stable homotopy theory was the characterization of infinite loop spaces, spaces that arise as the zero space of a spectrum. Following Boardman and Vogt [2], the approaches of May via $E_{\infty}$-operads [11] and Segal via $\Gamma$-spaces [15] provided characterizations of space-level data guaranteeing that a space possessed arbitrary deloopings. However, while in general the development of stable homotopy theory in the equivariant setting has been successful as in Lewis, May and Steinberger [7], the area of equivariant infinite loop space theory has remained mysterious.

In the nonequivariant setting, an infinite loop space is a space equipped with a multiplication which is commutative and associative up to all higher homotopies. The recognition principles explicitly encode this information-both $E_{\infty}$-operad actions and $\Gamma$-space structures are evidently devices for packaging up the coherent homotopies describing such a multiplication. Unfortunately, in the equivariant setting the structure carried by an infinite loop space is harder to understand. The additional complexity arises from the representation theory of the group $G$.

For certain applications, one can work with equivariant spectra which consist of a sequence of $G$-spaces $X_{n}$ and equivariant structure maps $S^{1} \wedge X_{n} \rightarrow X_{n+1}$ which induce $G$-equivalences $X_{n} \rightarrow \Omega X^{n+1}$. However, in order to have an equivariant version of Spanier-Whitehead duality, one has no choice but to work with spectra indexed on the collection of all finite-dimensional real representations of the group $G$ and equipped with structure maps for suspensions along such representations.

For a finite-dimensional real representation $V$, let $S^{V}$ denote the one-point compactification. A "genuine" equivariant spectrum $X$ has compatible structure maps $S^{W-V} \wedge X(V) \rightarrow X(W)$ which induce adjoint equivalences $X(V) \rightarrow \Omega^{W-V} X(W)$ for all pairs of finite-dimensional representations $V \subset W$, where $W-V$ is the orthogonal complement of $V$ in $W$. The zero space of such a spectrum carries a tremendous amount of structure inherited from the group $G$-it is a " $V$-fold" loop space for all finite-dimensional representations $V$.

Nonetheless, when $G$ is a finite group, straightforward generalizations of the nonequivariant recognition principles continue to apply. In the operadic setting, there is a notion of a $G$-operad and one can demonstrate that spaces that admit the action of an $E_{\infty}-G$-operad admit deloopings by arbitrary representation spheres; see Costenoble and Waner [3]. The equivariant argument follows the nonequivariant argument closely, and in particular depends on equivariant versions of the "approximation theorem", which describes models for the free loop spaces $\Omega^{V} S^{V} X$. Similarly, one can define an 
equivariant version of a $\Gamma$-space, and it can be shown that a "very special" equivariant $\Gamma$-space gives rise to a genuine $G$-spectrum; see Shimakawa [17] and Segal [16].

Unfortunately, serious difficulties arise in trying to generalize to the situation in which $G$ is an infinite compact Lie group. There is a simple counterexample due to Segal which demonstrates that the equivariant version of the approximation theorem fails, even for $G=S^{1}$ [16]. There are problems in trying to generalize the $\Gamma$-space approach as well. In the appendix, we will recall the counterexample of Segal and also analyze the failure of equivariant $\Gamma$-spaces to be a model for the equivariant stable homotopy category for any infinite compact Lie group $G$.

The intent of this paper is to begin to characterize the kind of structure that arises on the zero spaces of genuine equivariant spectra by studying the closely related category of equivariant continuous functors. This approach is suggested by the strategy of Segal's analysis of $\Gamma$-spaces. Recall that Segal proved that $\Gamma$-spaces describe infinite loop spaces by showing that a $\Gamma$-space gives rise via prolongation to a continuous functor from finite CW-complexes to based spaces which takes (most) homotopy pushouts to homotopy pullbacks [15]. Let $\mathscr{T}$ denote the category of compactly generated based spaces and $\mathscr{W}$ denotes the full subcategory of $\mathscr{T}$ consisting of spaces homeomorphic to finite CW-complexes. A continuous functor $X: \mathscr{W} \rightarrow \mathscr{T}$ is one for which the induced map of hom spaces

$$
\mathscr{W}(A, B) \stackrel{=}{\longrightarrow} \mathscr{T}(A, B) \stackrel{X}{\longrightarrow} \mathscr{T}(X(A), X(B))
$$

is continuous. A continuous functor $X$ between pointed categories is based if $X(*)=*$. We will assume herein that all continuous functors are based. It is reasonable to think of a continuous functor as a kind of spectrum because continuity implies the existence of a "structure map" $X(A) \wedge B \rightarrow X(A \wedge B)$. Indeed, in the nonequivariant setting Lydakis showed that a simplicial version of this category was a convenient symmetric monoidal category of spectra [8]. When a continuous functor $X$ takes homotopy pushouts to homotopy pullbacks, then the prespectrum $\left\{X\left(S^{n}\right)\right\}$ is an $\Omega$-prespectrum. Applying $X$ to the diagram

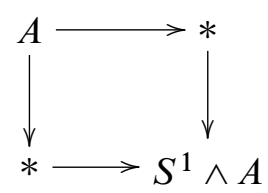

induces a weak equivalence between $X(A)$ and $\Omega X(\Sigma A)$. Therefore, we are led to seek the equivariant generalization of this property. In order to make this question precise, we need to specify exactly what we mean by an equivariant prespectrum. 
Fix a universe $U$, by which we mean an infinite (countable) dimensional real vector space $U$ on which $G$ acts by isometries and which is the direct sum of its finitedimensional $G$-invariant subspaces. We will assume that $U$ contains a trivial representation and each of its finite-dimensional subrepresentations infinitely often. The universe $U$ is complete if it contains all irreducible representations of $G$.

Remark 1.1 In the body of the paper, for simplicity we will assume that the universes we work with are complete. The results of the paper, suitably modified, remain true for incomplete universes. The specific modifications necessary and related subtleties which arise in the context of incomplete universes are discussed in Section 3.4.

A $G$-prespectrum is a collection of spaces $X(V)$ for finite-dimensional $V \in U$ equipped with compatible structure maps $S^{W-V} \wedge X(V) \rightarrow X(W)$. An $\Omega-G-$ prespectrum is a $G$-prespectrum $X$ with adjoint structure maps $X(V) \rightarrow \Omega^{W-V} X(W)$ which are $G$-equivalences.

Thus, for a compact Lie group $G$ we wish to know when a continuous functor $Z$ from finite $G$-CW-complexes to $G$-spaces yields a collection of spaces $\{Z(V)\}$ for $Z \in U$ which specifies a genuine equivariant $\Omega$-prespectrum indexed on the universe $U$. It is not sufficient for such a $Z$ to take $G$-homotopy pullbacks to $G$-homotopy pushouts, as the structure maps for nontrivial representations $V$ cannot be constructed in the above fashion.

Denote the category of based $G$-spaces that are homeomorphic to finite $G-\mathrm{CW}-$ complexes by $\mathscr{W}_{G}$. Note that we include all maps as morphisms, not just the equivariant maps. As a consequence, $\mathscr{W}_{G}$ is enriched over based $G$-spaces with the action on the morphism spaces given by conjugation. In analogy with the nonequivariant terminology, we say a functor from $\mathscr{W}_{G}$ to based $G$-spaces as continuous if the induced map of enriched hom $G$-spaces is a continuous map of $G$-spaces. We will refer to a based continuous functor from $\mathscr{W}_{G}$ to based $G$-spaces as a $\mathscr{W}_{G}$-space.

The main result of this paper is the following theorem, which implies that the additional sufficient condition for a $\mathscr{W}_{G}$-space $Z$ to represent an $\Omega-G$-prespectrum is a kind of compatibility with Spanier-Whitehead duality for the orbit spectra $\Sigma^{\infty} G / H_{+}$. Let $G / H$ be embedded in a real $G$-representation $V$, with normal bundle $v$. Denote by $T v$ the Thom space of $v$. Recall that there is a stable equivalence between $\Sigma^{\infty} G / H_{+}$ and $\Sigma_{V}^{\infty} T v\left(S^{-V} \wedge T v\right)$ as a consequence of Spanier-Whitehead duality. This stable duality is exhibited on the space level by a $V$-duality map $G / H_{+} \wedge T \nu \rightarrow S^{V}$.

Theorem 1.2 Let $G$ be a compact Lie group, $U$ a complete universe of $G$-representations, and $Z$ a $\mathscr{W}_{G}$-space. Let $A$ be any finite $G-C W-$ complex. Then the collection 
$\left\{Z\left(A \wedge S^{V}\right) \mid V \subset U\right\}$ is an $\Omega-G$-prespectrum if and only if (1) and either of the equivalent conditions (2) or $\left(2^{\prime}\right)$ hold.

(1) $Z$ takes $G$-homotopy pushout squares to $G$-homotopy pullback squares.

(2) Let $G / H$ be an orbit space embedded in a $G$-representation $V \subset U$, with normal bundle $v$. Let $T v$ denote the Thom space of $v$. For any $X \in \mathscr{W}_{G}$, a certain map

$$
Z(T v \wedge X) \rightarrow \operatorname{Map}_{0}\left(G / H_{+}, Z\left(S^{V} \wedge X\right)\right)
$$

is an equivalence. Here Map ${ }_{0}$ denotes the $G$-space of nonequivariant based maps, with $G$ acting by conjugation.

(2') Let $G / H$ be an orbit space embedded in a $G$-representation $V \subset U$. Let $L$ denote the tangent $H$-representation at the identity coset. For any $X \in \mathscr{W}_{H}$, a certain map

$$
Z\left(G_{+} \wedge_{H} X\right) \rightarrow \operatorname{Map}_{H}\left(G_{+}, Z\left(S^{L} \wedge X\right)\right)
$$

is an equivalence.

The maps in conditions (2) and $\left(2^{\prime}\right)$ are induced from the $V$-duality $T \nu \wedge G / H_{+} \rightarrow S^{V}$ and will be described precisely in Hypothesis 3.3. The second version of the orbit condition arises from the generalized Wirthmuller isomorphism [13; 7].

A based continuous functor $Z$ which satisfies either of the equivalent conditions above will be said to be "equivariantly linear with respect to $U$ ". This terminology is motivated by the fact that our notion of equivariantly linear provides a precise generalization of the linearity conditions of Mandell, May, Schwede and Shipley [10]. One might also describe such a functor $Z$ as "genuinely" equivariantly excisive, in line with the language of Goodwillie's calculus of functors. It is interesting to wonder if this notion is relevant to possible equivariant generalizations of the calculus of functors.

Before we move on, it is worth attempting to provide some intuition about why this compatibility with Atiyah duality for orbit spectra is a reasonable condition to expect, beyond what is provided by the details of the proof of the theorem. A related problem to the one we consider herein is to determine when a $\mathbb{Z}$-graded cohomology theory on $G$-spaces with coefficients in a coefficient system $M$ extends to an $R O(G)-$ graded cohomology theory. Such an extension exists if and only if $M$ extends to a Mackey functor [12, IX.5.2]. It is illuminating to recall the data that is required for such an extension. Essentially, the key observation is that the stable transfer maps $\tau(G / H): S^{V} \rightarrow(G / H)_{+} \wedge S^{V}$ and $\tau(\pi):(G / K)_{+} \wedge S^{V} \rightarrow(G / H)_{+} \wedge S^{V}$ (where $\pi$ is the projection $G / H \rightarrow G / K, H \subset K \subset G$ ) yield transfer homomorphisms in 
the cohomology theory, and these in turn give rise to transfers $M(G / H) \rightarrow M(G / K)$ and $M(G / H) \rightarrow M(G / G)$ respectively. The construction of these transfer maps is intimately connected to Atiyah duality for the orbit spectra $\Sigma^{\infty} G / H_{+}$; this relationship can be seen via an explicit construction in terms of Pontryagin-Thom maps [12, IX.3] or from the perspective of formal categorical duality [12, XVII.1].

There is a model theoretic interpretation of Theorem 1.2, obtained by situating the theorem in the context of the study of diagram spectra. The work of Lydakis [8] and Mandell, May, Schwede and Shipley [10] permits the following modern reinterpretation of the $\Gamma$-space approach to infinite loop space theory. Denote by $\mathscr{P}$ the category of prespectra and $\mathscr{F} \mathscr{T}$ the category of $\Gamma$-spaces. Let $\mathscr{W} \mathscr{T}$ be the category of continuous functors from $\mathscr{W}$ to based spaces. Our interest in $\mathscr{W} \mathscr{T}$ is its intermediate position between $\Gamma$-spaces and prespectra. These various categories of spectra are linked by adjoint pairs $(\mathbb{P}, \mathbb{U})$, where we regard the left adjoint $\mathbb{P}$ as prolongation and the right adjoint $\mathbb{U}$ is the restriction:

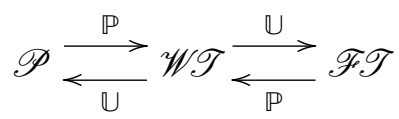

With suitable stable model structures, the first adjoint pair induces a Quillen equivalence and the second adjoint pair is a Quillen adjunction which induces an equivalence of the respective homotopy categories of connective objects (a "connective" Quillen equivalence [10]). From this standpoint, the work of Segal [15] amounts to the proof that for a "very special" $\Gamma$-space $E$, the $\mathscr{W}$-space $\mathbb{P} E$ is fibrant and therefore the prespectrum $\mathbb{U P} E$ is fibrant and hence an $\Omega$-prespectrum.

To understand the situation equivariantly, it is natural to ask how much of this analysis can be generalized. When $G$ is finite, one can obtain an identical version of this diagram. The homotopical analysis of equivariant $\Gamma$-spaces is known $[16 ; 17]$ and we intend to discuss the model theoretic aspects of this elsewhere. When $G$ is an infinite compact Lie group, even though we cannot hope to have a "connective" equivalence of the equivariant analogues of $\mathscr{W}$-spaces and $\Gamma$-spaces, a concrete understanding of the lefthand terms in the comparison diagram should indicate the nature of the generalization of $\Gamma$-spaces that will be needed.

We begin by carrying out an essentially formal reworking of the model category theory associated to $\mathscr{W}$-spaces [10] in the equivariant setting. Let $G \mathscr{W} \mathscr{T}$ denote the category of $\mathscr{W}_{G}$-spaces with morphisms the $G$-equivariant natural transformations. Fix a complete universe $U$ of real $G$-representations.

We have an adjoint pair of functors $(\mathbb{P}, \mathbb{U})$ connecting the category of $G$-prespectra indexed on $U$ and the category of $\mathscr{W}_{G}$-spaces, where the left adjoint $\mathbb{P}$ is a prolongation 
and the right adjoint $\mathbb{U}$ is a restriction:

$$
G \mathscr{P} \underset{\mathbb{U}}{\stackrel{\mathbb{P}}{\rightleftarrows}} G \mathscr{W} \mathscr{T}
$$

This allows us to define the homotopy groups of a $\mathscr{W}_{G}$-space $Z$ as the equivariant homotopy groups of the associated prespectrum $\cup Z$. That is, $\pi_{*}(Z)=\pi_{*} \mathbb{U} Z$, where the homotopy groups run over fixed-point spaces corresponding to all closed subgroups of $G$ [9, 3.3.2]. In addition, $G \mathscr{W} \mathscr{T}$ is symmetric monoidal with a smash product constructed by Kan extension [9, 2.3.1]. The comparison to $G$-prespectra factors through the category of orthogonal $G$-spectra (denoted $G \mathscr{I S}$ ) via another adjoint pair $(\mathbb{P}, \mathbb{U})$

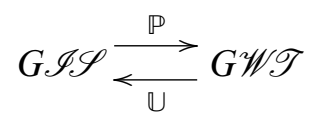

where $\mathbb{P}$ and $\mathbb{U}$ are respectively strong and lax symmetric monoidal. With this framework, we prove the following theorem recapitulating the nonequivariant theory of $\mathscr{W}$-spaces [10, 17.1-17.6] in the equivariant setting. Note that a " $G$-topological" model structure satisfies an appropriate analogue of Quillen's axiom SM7 reflecting compatibility with the $G$-enrichment $[9,3.1 .4]$.

Theorem 1.3 Fix a complete $G$-universe $U$.

(1) There is a cofibrantly generated $G$-topological model structure on the category $G \mathscr{W} \mathscr{T}$ in which the weak equivalences are the $\pi_{*}$-equivalences, the "stable model structure".

(2) The fibrant objects in the stable model structure on $G \mathscr{W} \mathscr{T}$ are the $\mathscr{W}_{G}$-spaces $Z$ such that $\mathbb{U} Z$ is an $\Omega-G$-prespectrum.

(3) The stable model structures satisfies the monoid and pushout-product axioms with respect to the smash product, and hence can be lifted to a model structure on categories of rings and modules.

(4) The adjoint pair $(\mathbb{P}, \mathbb{U})$ connecting $G \mathscr{W} \mathscr{T}$ to $G \mathscr{I S}$ is a Quillen equivalence.

(5) There is a different model structure on $G \mathscr{W} \mathscr{T}$ which is a cofibrantly generated $G$-topological model structure in which the weak equivalences are the $\pi_{*}-$ equivalences, the "absolute stable model structure". The identity functor is the left adjoint of a Quillen equivalence between the stable model structure and the absolute stable model structure.

(6) The fibrant objects in the absolute stable model structure on $G \mathscr{W} \mathscr{T}$ are the $\mathscr{W}_{G}$-spaces $Z$ such that the collection $\left\{Z\left(A \wedge S^{V}\right)\right\}$ as $V$ varies over $U$ is an $\Omega-G$-prespectrum for any $A \in \mathscr{W}_{G}$. 
By itself, this formal analysis has not bought us very much new information. However, let us again recall the nonequivariant situation. There, one proves that the prolongation of a "very special" $\Gamma$-space $X$ yields a $\mathscr{W}$-space $\tilde{X}$ which is fibrant in the absolute stable model structure. A $\mathscr{W}$-space which is fibrant in the absolute stable model structure is clearly fibrant in the stable model structure as well, and then the restriction $\mathbb{U} \tilde{X}$ is a fibrant prespectrum. It is important to be clear about the roles of the two stable model structures on $\mathscr{W}$-spaces. Although at the end of the day we are interested in fibrant objects in the stable model structure on $\mathscr{W}$-spaces, the absolute stable model structure is essential in order to compare $\mathscr{W}$-spaces to $\Gamma$-spaces. In addition, it turns out that the fibrant objects in the absolute stable model structure admit a concise intrinsic description in the nonequivariant setting - they are precisely the linear functors.

Thus, we are led to the question of determining a similar intrinsic description of the $\mathscr{W}_{G}$-spaces $Z$ which are fibrant in the absolute stable model structure. Explicitly, we want conditions which are necessary and sufficient for the prespectra $\left\{Z\left(A \wedge S^{V}\right)\right\}$ to be $\Omega-G$-prespectra. Such conditions are provided by Theorem 1.2.

Corollary 1.4 A $\mathscr{W}_{G}$-space is fibrant in the absolute stable model structure on $G \mathscr{W} \mathscr{T}$ if and only if it is equivariantly linear.

The concrete characterization of equivariantly linear functors provides an indication of the information that must be captured by a space-level recognition principle for equivariant infinite loop spaces. For instance, it suggests that the appropriate equivariant analogue of $\Gamma$-spaces involve an enlarged domain category which contains all the orbit spaces $G / H$. In future work, we intend to exploit this perspective.

The paper is organized as follows. In section 2, we briefly state the definitions and model theoretic results we will refer to in the course of proving the main theorem. We relegate proofs to the appendix. In section 3, we prove Theorem 1.2. In the first section of the appendix, we carry out the model theoretic analysis of the category of $\mathscr{W}_{G}-$ spaces. This is very similar to the analysis of [10], and our primary purpose is to record results and the proofs of supporting lemmas which do not follow immediately from the nonequivariant results. In the second part of the appendix, we analyze $\Gamma-S^{1}$-spaces.

Acknowledgements I wish to express my gratitude to Peter May for proposing this area of research, for many useful discussions, and for careful reading of previous drafts. I would also like to thank Mike Mandell for his invaluable assistance and suggestions. Brooke Shipley and Chris Douglas provided valuable comments based on an earlier draft, and I had helpful conversations with Mark Behrens, Ben Lee, and Ben Wieland. In particular, I would like to thank Ben Wieland for catching a mistake in a previous version of the paper. The exposition of this paper was significantly improved by comments from an anonymous referee. 


\section{Basic definitions and a rapid overview of model theoretic results}

In this section, we will present the basic definitions and summarize the model theoretic results. The proofs of these results appears in the first section of the appendix.

\subsection{Categories of $\mathscr{W}_{G}-$ spaces}

The categories we will be working with are enriched over based $G$-spaces. Thus, our discussion could be cast entirely in terms of enriched category theory. However, we follow the convention of Mandell and May [9] and instead consider ordinary functors with additional conditions in order to emphasize the analogies to the nonequivariant case and to minimize overhead. As a consequence, we will work with pairs $\mathscr{C}_{G}$ and $G \mathscr{C}=\left(\mathscr{C}_{G}\right)^{G}$, where $\mathscr{C}_{G}$ is a category of $G$-objects and nonequivariant maps between them, and $G \mathscr{C}$ is obtained by restricting to the $G$-maps. The hom spaces of $\mathscr{C}_{G}$ are given a $G$-action via conjugation, and regarded as based via the addition of a $G$-fixed basepoint as necessary. Therefore, we can obtain the space of $G$-maps by taking fixed points. For instance, let $\mathscr{T}_{G}$ be the category of based $G$-spaces with morphisms all maps of nonequivariant spaces. Then $\operatorname{hom}_{\mathscr{T}_{G}}(X, Y)$ has a $G$-action given by conjugation. The category $G \mathscr{T}$ is obtained by passage to $G$-fixed points on the hom spaces, and is the category of $G$-spaces and $G$-maps. All of the model structures we consider are compatible with this enrichment; we will refer to such model categories as $G$-topological. The precise definition of this compatibility is discussed in the appendix.

With this in mind, we can now define the categories of $\mathscr{W}_{G}$-spaces we will be working with. Recall that $\mathscr{W}_{G}$ denotes the category of based $G$-spaces homeomorphic to finite $G-\mathrm{CW}$-complexes, with morphisms all continuous (but not necessarily equivariant) maps. This is a full subcategory of $\mathscr{T}_{G}$. Although $\mathscr{W}_{G}$ is not small, it is skeletally small and throughout we will tacitly assume that we have chosen a small skeleton of $\mathscr{W}_{G}$ and are working relative to that skeleton.

Definition 2.1 The category $\mathscr{W}_{G} \mathscr{T}$ has as objects the based continuous $G$-functors from $\mathscr{W}_{G}$ to $\mathscr{T}_{G}$. The morphisms are the natural transformations between functors. We can topologize the morphisms as a subspace of the product of the function spaces $\operatorname{Map}_{0}(X(A), Y(A))$ over $A \in \mathscr{W}_{G}$, which has a $G$-action by conjugation.

The category $G \mathscr{W} \mathscr{T}$ of $\mathscr{W}_{G}$-spaces is obtained by passage to $G$-fixed points from $\mathscr{W}_{G} \mathscr{T}$. That is, the objects are again the continuous $G$-functors from $\mathscr{W}_{G}$ to $\mathscr{T}_{G}$ and the morphisms are the natural $G$-maps.

Just as in the case of orthogonal $G$-spectra $[9,2.3 .1]$, we have the following theorem. 
Theorem 2.2 The categories $\mathscr{W}_{G} \mathscr{T}$ and $G \mathscr{W} \mathscr{T}$ have smash product and function spectrum functors which make them closed symmetric monoidal categories. The unit is the identity functor.

The smash product is constructed in the usual way as an internalization of the obvious external smash product via left Kan extension. Some care has to be taken to verify that the Kan extension exists [9, 2.6.7].

\subsection{The stable model structure}

Throughout, fix a complete universe $U$. The first model structure we consider is the relative level model structure on $G \mathscr{W} \mathscr{T}$, where by relative we mean that the fibrations and weak equivalences are detected only on the spheres $\left\{S^{V}\right\}$ for $V \in U$.

Definition 2.3 The relative level model structure on this category is defined as follows. A map $Y \rightarrow Z$ is

(1) a fibration if each $Y\left(S^{V}\right) \rightarrow Z\left(S^{V}\right)$ is an equivariant Serre fibration,

(2) a weak equivalence if each $Y\left(S^{V}\right) \rightarrow Z\left(S^{V}\right)$ is an equivariant weak equivalence,

(3) a cofibration if it has the left-lifting property with respect to the acyclic fibrations.

Proposition 2.4 The relative level model structure on $G \mathscr{W} \mathscr{T}$ is a cofibrantly generated $G$-topological model structure.

There is an associated stable model structure. We define $\pi_{*} Z$ for a $\mathscr{W}_{G}-$ space $Z$ by passing to the $G$-prespectrum $\mathbb{U} Z$ and specifying $\pi_{*} Z=\pi_{*} \cup Z$. Recall that for a subgroup $H$ of $G$ and an integer $q$, for $q \geq 0$ we define

$$
\pi_{q}^{H}(\mathbb{U} Z)=\operatorname{colim}_{V} \pi_{q}^{H}\left(\Omega^{V} Z(V)\right)
$$

and for $q>0$ we define

$$
\pi_{-q}^{H}(\mathbb{U} Z)=\operatorname{colim}_{V \supseteq \mathbb{R}^{q}} \pi_{0}^{H}\left(\Omega^{V-\mathbb{R}^{q}} Z(V)\right) .
$$

These equivariant homotopy groups capture stable equivalences.

Definition 2.5 Let $Z_{1}$ and $Z_{2}$ be $\mathscr{W}_{G}$-spaces. A map $f: X_{1} \rightarrow X_{2}$ is a $\pi_{*}-$ isomorphism if the induced maps $f_{*}: \pi_{q}^{H}\left(X_{1}\right) \rightarrow \pi_{q}^{H}\left(X_{2}\right)$ are isomorphisms for all closed subgroups $H \subset G$. 
Definition 2.6 In the stable model structure, a map is

(1) a cofibration if it is a cofibration in the relative level model structure,

(2) a weak equivalence if it is a $\pi_{*}$-equivalence,

(3) a fibration if it has the right-lifting property with respect to the acyclic cofibrations (maps which are both level cofibrations and $\pi_{*}$-equivalences).

Proposition 2.7 The stable model structure is a $G$-topological model structure on the category $G \mathscr{W} \mathscr{T}$.

There is a pair of adjoint functors $(\mathbb{P}, \mathbb{U})$ connecting $G \mathscr{W} \mathscr{T}$ and the category $G \mathscr{I S}$ of orthogonal $G$-spectra $[9 ; 10] . \mathbb{U}$ is the forgetful functor from $\mathscr{W}_{G}$-spaces to orthogonal $G$-spectra, and $\mathbb{P}$ is the prolongation constructed as a left Kan extension along the inclusion of domain categories [10, 23.1]. We have the expected comparison result.

Theorem 2.8 The pair $(\mathbb{P}, \mathbb{U})$ specifies a Quillen equivalence between the stable model category structure on $G \mathscr{W} \mathscr{T}$ and the stable model category structure on $G \mathscr{I S}$.

\subsection{The absolute stable model structure}

The level model structure used to construct the stable model structure in the previous section depends on evaluation at the spheres. This makes it inconvenient to compare to diagram categories where the domain does not include an embedding of the spheres, for instance $\Gamma-G$-spaces. As in the nonequivariant case, we rectify this by considering an "absolute" model structure.

Definition 2.9 The absolute level model structure on $G \mathscr{W} \mathscr{T}$ is defined as follows. A map $Y \rightarrow Z$ is

(1) a fibration if each $Y(A) \rightarrow Z(A)$ for $A \in \mathscr{W}_{G}$ is an equivariant Serre fibration,

(2) a weak equivalence if each $Y(A) \rightarrow Z(A)$ for $A \in \mathscr{W}_{G}$ is an equivariant weak equivalence,

(3) a cofibration if it has the left-lifting property with respect to the acyclic fibrations.

There is an associated absolute stable model structure. 
Definition 2.10 In the absolute stable model structure, a map is

(1) a cofibration if it is a cofibration in the absolute level model structure,

(2) a weak equivalence if it is a $\pi_{*}$-equivalence,

(3) a fibration if it has the right-lifting property with respect to the acyclic cofibrations.

Proposition 2.11 The stable model structure is a $G$-topological model structure on the category $G \mathscr{W} \mathscr{T}$.

In the course of proving the previous proposition, we obtain an identification of the fibrant objects in the absolute stable model structure.

Proposition 2.12 A $\mathscr{W}_{G}$-space $Z$ is fibrant in the absolute stable model structure if and only if for all $A \in \mathscr{W}_{G}$ and $W \in U$ the structure map

$$
Z(A) \rightarrow \Omega^{W} Z\left(S^{W} \wedge A\right)
$$

is a weak equivalence.

There is a Quillen equivalence between the relative stable model structure and the absolute stable model structure.

Theorem 2.13 The identify functor is the left adjoint of a Quillen equivalence between the category of $\mathscr{W}_{G}$-spaces with the relative stable model structure and the category of $\mathscr{W}_{G}$-spaces with the absolute stable model structure.

\subsection{Ring and module spectra}

Just as in the nonequivariant setting, we can lift the stable model structure to categories of ring and module $\mathscr{W}_{G}$-spaces. While we do not require this in the paper, it is a useful feature of this perspective on the equivariant stable category.

Theorem 2.14 Let $R$ be a ring $\mathscr{W}_{G}$-space.

(1) The category of $R$-module $\mathscr{W}_{G}$-spaces is a cofibrantly generated proper $G-$ topological model category, with weak equivalences and fibrations created in the stable model structure on the category of $\mathscr{W}_{G}$-spaces.

(2) If $R$ is commutative, the category of $R$-algebra $\mathscr{W}_{G}$-spaces is a cofibrantly generated right proper $G$-topological model category with weak equivalences and fibrations created in the stable model structure on the category of $\mathscr{W}_{G}$-spaces.

Remark 2.15 The obvious variant of this theorem starting from the absolute stable model structure also holds. 


\section{Continuous $G-$ functors and $\Omega-G$-prespectra}

In this section we will provide concrete conditions which describe the fibrant objects in the absolute stable model structure on $\mathscr{W}_{G}$-spaces. That is, we specify conditions on a $\mathscr{W}_{G}$-space $Z$ which guarantee that for any $A \in \mathscr{W}_{G}$, the prespectrum obtained as the collection of spaces $\left\{Z\left(S^{V} \wedge A\right)\right\}$ is a genuine $\Omega-G$-spectrum. These conditions amount to enforcing a suitable interaction with equivariant Spanier-Whitehead duality (or more precisely equivariant Atiyah duality) for orbit spectra. This connection to duality highlights the difficulty of generalizing recognition principles from the case of $G$ finite to the case of $G$ a compact Lie group, for only when $G$ is finite are the orbit spectra self dual.

\subsection{Linearity in the nonequivariant setting}

First, we recall the nonequivariant situation. Let $Z$ be a continuous functor from $\mathscr{W}$ to spaces. There is a structure map $\sigma: Z(A) \wedge B \rightarrow Z(A \wedge B)[10,4.9]$ which arises as a consequence of continuity. The map $\sigma$ is the adjoint of the composite

$$
B \stackrel{\alpha}{\longrightarrow} \mathscr{T}(A, A \wedge B) \stackrel{=}{\longrightarrow} \mathscr{W}(A, A \wedge B) \stackrel{Z}{\longrightarrow} \mathscr{T}(X(A), X(A \wedge B)),
$$

where $\alpha(b)(a)=a \wedge b$. Setting $B=S^{n}$, this gives us the structure maps of a prespectrum when we consider the collection $\left\{Z\left(S^{n}\right)\right\}$. We will denote this prespectrum by $Z\left[S^{0}\right]$, and write $Z[A]$ for the prespectrum $\left\{Z\left(S^{n} \wedge A\right)\right\}$. Observe that $Z\left[S^{0}\right]=$ $\mathbb{U} Z$. Also, note that the structure maps $Z(A) \wedge I_{+} \rightarrow Z\left(A \wedge I_{+}\right)$imply that any $\mathscr{W}$-space preserves homotopies and hence weak equivalences on $\mathscr{W}[10,17.4]$.

It is well known that there is a simple condition on $Z$ which guarantees that $Z[A]$ is an $\Omega$-prespectrum. For this to be true, it must be the case that $Z$ takes homotopy pushout squares to homotopy pullback squares. This is sometimes stated as $Z$ is linear. Given such a $Z$, for any $A \in \mathscr{W}$ we have the homotopy pushout

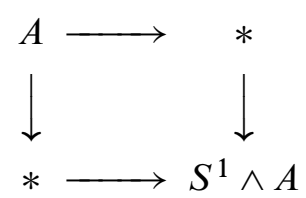

which constructs the suspension, and when we apply $Z$ to this diagram there is an induced weak equivalence between $Z(A)$ and $\Omega Z(\Sigma A)$.

We now wish to generalize this to describe similar conditions in the case of a $\mathscr{W}_{G}$-space $Z$ which will guarantee that the collection $\left\{Z\left(S^{V} \wedge A\right)\right\}$ forms an $\Omega-G$-spectrum. 
Denote the prespectrum $\left\{Z\left(S^{V} \wedge A\right)\right\}$ by $Z_{U}[A]$. Again, note that $Z_{U}\left[S^{0}\right]=\mathbb{U} Z$. The structure maps arise via an adjunction analogous to the nonequivariant case:

$$
B \stackrel{\alpha}{\longrightarrow} \mathscr{T}_{G}(A, A \wedge B) \stackrel{=}{\longrightarrow} \mathscr{W}_{G}(A, A \wedge B) \stackrel{Z}{\longrightarrow} \mathscr{T}_{G}(Z(A), Z(A \wedge B))
$$

Once again, the existence of these structure maps implies that $\mathscr{W}_{G}$-spaces preserve weak equivalences in $\mathscr{W}_{G}$. Next, observe that taking homotopy pushout squares to homotopy pullback squares (in the category of $G$-spaces) is insufficient to handle desuspension by arbitrary representations. We cannot construct $\Omega^{V} Z\left(S^{V}\right)$ for arbitrary representations $V$ in the fashion above. This condition is however enough to construct a naive $\Omega-G$ spectrum (indexed on a trivial universe).

Following [10, 17.9], we obtain the following characterization of continuous functors which generate naive $\Omega-G$-prespectra.

Proposition 3.1 Let $Z$ be a $\mathscr{W}_{G}$-space. The following are equivalent:

(1) $Z$ takes $G$-homotopy pushout squares to $G$-homotopy pullback squares.

(2) For any $A \in \mathscr{W}_{G}$, the prespectrum $Z[A]$ is a naive $\Omega-G$-prespectrum.

(3) For any $A \in \mathscr{W}_{G}$, the adjoint structure map $Z(A) \rightarrow \Omega Z(\Sigma A)$ is an equivalence.

We will call such $\mathscr{W}_{G}$-spaces "naively equivariantly linear". In order to handle suspensions at arbitrary representations, we need to specify more data about the functor $Z$.

\subsection{Compatibility with equivariant Spanier-Whitehead duality}

Fix a complete universe $U$. Let $V$ be a $G$-representation in $U$ and let $G / H$ be an orbit $G$-space which is embedded in $V$. Denote by $T v$ the Thom space of the normal bundle $v$ of the embedding, and note that this is $G$-homeomorphic to the compactification of a tubular neighborhood of $G / H$ because $G / H$ is compact. More concretely, for sufficiently small $\epsilon$ we can describe the tubular neighborhood of $G / H$ in $V$ as $(G / H)_{\epsilon}$, the $\epsilon$-neighborhood of $G / H$. Then $T v$ is $G$-homeomorphic to $(G / H)_{\epsilon}^{c}$, the one-point compactification of $(G / H)_{\epsilon}$. We know that $G / H_{+}$and $(G / H)_{\epsilon}^{c}$ are equivariantly $V$-dual.

This duality can be exhibited by the following map. There is a map

$$
G / H \rightarrow \operatorname{Map}\left(D(\epsilon),(G / H)_{\epsilon}\right)
$$

taking each $m \in G / H$ to the map which takes an element $x$ of the $\epsilon$-ball $D(\epsilon)$ about the origin to $m+x$. This induces a based map

$$
(G / H)_{+} \rightarrow \operatorname{Map}_{0}\left((G / H)_{\epsilon}^{c}, D(\epsilon)^{c}\right) \cong \operatorname{Map}_{0}\left((G / H)_{\epsilon}^{c}, S^{V}\right)
$$


by taking an element of $(G / H)_{\epsilon}^{c}$ to the basepoint if it is not within $\epsilon$ of $m$ and to $x-m$ otherwise. The adjoint of this is the duality map

$$
G / H_{\epsilon}^{c} \wedge(G / H)_{+} \rightarrow S^{V} .
$$

This is the classical Atiyah duality map. Note that we don't actually use the fact that $G / H$ is a submanifold of $V$. This map makes sense whenever we have a compact $G$-subset of $V$, and is the duality map when $\epsilon$ is sufficiently small.

Now assume that we have a $\mathscr{W}_{G}-$ space $Z$. Given the map

$$
(G / H)_{+} \rightarrow \operatorname{Map}_{0}\left((G / H)_{\epsilon}^{c}, S^{V}\right)
$$

by functoriality we obtain a map

$$
(G / H)_{+} \rightarrow \operatorname{Map}_{0}\left(Z\left((G / H)_{\epsilon}^{c}\right), Z\left(S^{V}\right)\right)
$$

and by adjunction we have a map

$$
\xi: Z\left((G / H)_{\epsilon}^{c}\right) \rightarrow \operatorname{Map}_{0}\left((G / H)_{+}, Z\left(S^{V}\right)\right) .
$$

Now, if we have a space $X \in \mathscr{W}_{G}$ and smash the duality map on both sides by $X$, by the same process we obtain a map

$$
\xi: Z\left((G / H)_{\epsilon}^{c} \wedge X\right) \rightarrow \operatorname{Map}_{0}\left((G / H)_{+}, Z\left(S^{V} \wedge X\right)\right) .
$$

Remark 3.2 One subtlety of the duality theory developed by Lewis, May and Steinberger [7,3.1-3.8] is that for given $V$-duals $X$ and $Y$ there are many possible choices of space-level maps exhibiting the $V$-duality. In the specific case of $G / H$ (and more generally for embedded submanifolds), there is another very explicit description of the duality between $G / H_{+}$and $T \nu$, the Thom space of the normal bundle of the embedding.

Specifically, we can construct a map

$$
T \nu \wedge G / H_{+} \rightarrow G / H_{+} \wedge S^{V} \rightarrow S^{V}
$$

where the first map is a Pontryagin-Thom map associated with a tubular neighborhood of the composite

$$
G / H \rightarrow G / H \times G / H \rightarrow v \times G / H
$$

and the second is the collapse map of $G / H_{+}$onto $S^{0}[7,3.5 .1]$. By functoriality and manipulation of adjoints we can obtain a map

$$
\xi_{2}: Z(T v) \rightarrow \operatorname{Map}_{0}\left(G / H_{+}, Z\left(S^{V}\right)\right)
$$

analogously to the construction of $\xi$. 
Under the homeomorphism given by the tubular neighborhood theorem $T v \rightarrow G / H_{\epsilon}^{c}$, the maps $\xi$ and $\xi_{2}$ coincide [7, 3.5.1]. As a consequence, our conditions below could be phrased in terms of the map $\xi_{2}$ rather than $\xi$, and in general, we could phrase them abstractly in terms of any system of duality maps which are suitably functorial.

We are now ready to formulate the first version of the additional conditions required for a $\mathscr{W}_{G}-$ space to represent a genuine $\Omega-G$-prespectrum. We refer to the condition below as "hypothesis (A)".

Hypothesis 3.3 A $\mathscr{W}_{G}$-space $Z$ satisfies hypothesis (A) for the universe $U$ if the following two conditions hold.

(1) $Z$ takes $G$-homotopy pushout squares to $G$-homotopy pullback squares.

(2) For all $X \in \mathscr{W}_{G}$ and any smooth embedding $G / H \longleftrightarrow V, V \in U$, there exists $\epsilon>0$ such that the map

$$
\left.\xi: Z\left((G / H)_{\epsilon}^{c}\right) \wedge X\right) \rightarrow \operatorname{Map}_{0}\left(G / H_{+}, Z\left(S^{V} \wedge X\right)\right)
$$

is a $G$-equivalence.

We will need a lemma extending this condition slightly.

Lemma 3.4 If $Z$ satisfies hypothesis (A) and $G / H$ embeds in $U$, then for any smooth embedding $G / H \times D^{n} \longleftrightarrow V$, there exists $\epsilon>0$ such that the map

$$
\left.\xi: Z\left(\left(G / H \times D^{n}\right)_{\epsilon}^{c}\right) \wedge X\right) \rightarrow \operatorname{Map}_{0}\left(G / H_{+}, Z\left(S^{V} \wedge X\right)\right)
$$

is a $G$-equivalence.

First, we show that this condition is sufficient. The argument below is an adaptation of the argument due to Segal [16] (and corrected by Shimakawa [17]) for the case when $G$ is a finite group. Recall that we wrote $Z_{U}[A]$ to refer to the prespectrum $\left\{Z\left(S^{V} \wedge A\right)\right\}$.

Theorem 3.5 Let $Z$ be a $\mathscr{W}_{G}$-space which satisfies hypothesis (A) for the universe $U$. Then $Z_{U}[A]$ is an $\Omega-G$-prespectrum.

Proof Fix an arbitrary representation $V$. By naive linearity, without loss of generality we can assume that $V$ contains a trivial representation $\mathbb{R}$. This assumption allows us to provide $G$-fixed basepoints to subspaces of $V$. Let $D(1)$ denote the unit disk in 
$V$, and $S(1)$ the unit sphere which is the boundary, and in general let $D(r)$ and $S(r)$ be the disk and its boundary of radius $r$. There is a commutative diagram

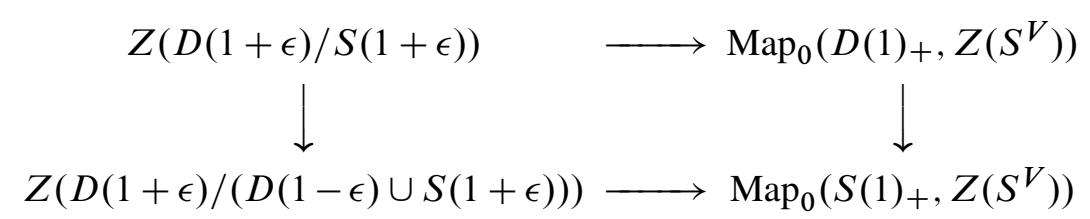

where the horizontal maps are defined analogously to the map $\xi$.

The top horizontal map is clearly an equivalence, and temporarily assume the bottom horizontal map is an equivalence. The right vertical map is a fibration induced from the inclusion $S(1)_{+} \hookrightarrow D(1)_{+}$, and the fiber is $\Omega^{V} Z\left(S^{V}\right)$. There is a cofibration sequence

$(D(1-\epsilon) \cup S(1+\epsilon)) / S(1+\epsilon) \rightarrow D(1+\epsilon) / S(1+\epsilon) \rightarrow D(1+\epsilon) /(D(1-\epsilon) \cup S(1+\epsilon))$.

Since $(D(1-\epsilon) \cup S(1+\epsilon)) / S(1+\epsilon) \simeq S^{0}$ and $Z$ takes cofibration sequences to fibration sequences by hypothesis, we know that the homotopy fiber of the left vertical map is $Z\left(S^{0}\right)$. Therefore, we can conclude that there is an equivalence $Z\left(S^{0}\right) \simeq \Omega^{V} Z\left(S^{V}\right)$. The induced map of fibers from $Z\left(S^{0}\right) \rightarrow \Omega^{V} Z\left(S^{V}\right)$ is indeed the adjoint of the structure map, as it is obtained from the embedding of 0 in $V$. Since $Z\left(A \wedge S^{W} \wedge-\right)$ is also a functor satisfying our hypotheses, we obtain the desired equivalence $Z\left(S^{W} \wedge A\right) \simeq \Omega^{V} Z\left(S^{V \oplus W} \wedge A\right)$ for every $A \in \mathscr{W}_{G}$.

Therefore, to complete the proof of the theorem it will suffice to verify that the bottom map is indeed a weak equivalence. As an aside, note that the bottom map can be described as the map $\xi_{2}: Z(T v) \rightarrow \operatorname{Map}_{0}\left(S(1)_{+}, Z\left(S^{V}\right)\right)$, where $T v$ here is Thom space of the normal bundle of the embedding of $S(1)$ in $V$.

Since $S(1)$ is a finite $G-\mathrm{CW}-$ complex, we proceed by induction. We can decompose $S(1)$ as a regular $G-\mathrm{CW}-$ complex [5]. That is, we can regard it as comprised of cells $G / H \times D^{n}$ (for varying $H$ ) where the attaching maps are homeomorphisms and the images of the boundary $G / H \times S^{n-1}$ are equal to unions of cells of lower dimension. Moreover, by subdividing if necessary, we can assume that the closed cells $G / H \times D^{n}$ are subcomplexes. We will fix a choice of homeomorphic embedding of $S(1)$ in $V$. Recall that $D^{n}$ has trivial $G$-action.

Let $X$ be a subcomplex of $S(1)$, a union of some of the cells of $S(1)$. We have maps

$$
\xi_{X}: Z\left(X_{\epsilon}^{c}\right) \rightarrow \operatorname{Map}_{0}\left(X_{+}, Z\left(S^{V}\right)\right)
$$

which we can regard as induced by restriction of the map

$$
S(1) \rightarrow \operatorname{Map}_{0}\left(D(\epsilon), S(1)_{\epsilon}\right)
$$

Algebraic $8 \mathcal{G}$ Geometric Topology, Volume 6 (2006) 
used to construct $\xi$.

We will induct downward over the number of cells in $X$. The base cases therefore involve $X$ consisting of a single cell $G / H \times D^{n}$. In this situation, the map $\xi_{X}$ is an equivalence by Lemma 3.4.

Let the number of cells in $X$ be $m$, and assume that $\xi$ is an equivalence for subcomplexes with $m-1$ cells or fewer. Let $G / H \times D^{n}$ be a cell of highest dimension in $X$, and let $Y$ be the union of the remaining cells, so that $X=Y \cup\left(G / H \times D^{n}\right)$.

There is a commutative diagram

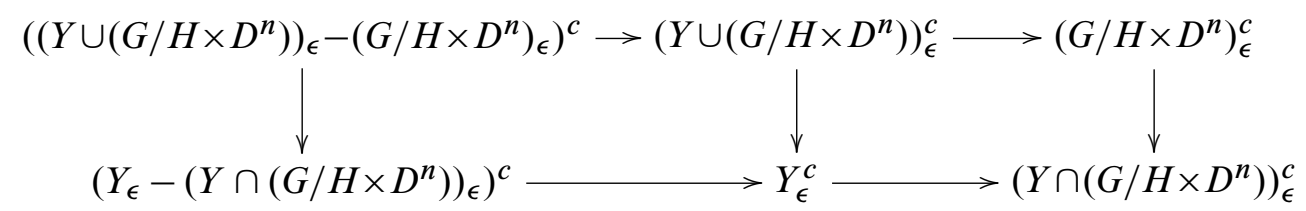

where each row is a cofibration. The map

$$
\left(\left(Y \cup\left(G / H \times D^{n}\right)\right)_{\epsilon}-\left(G / H \times D^{n}\right)_{\epsilon}\right)^{c} \rightarrow\left(Y_{\epsilon}-\left(Y \cap\left(G / H \times D^{n}\right)\right)_{\epsilon}\right)^{c}
$$

is a weak equivalence, as follows. Recall that for $\epsilon$ sufficiently small we can naturally replace the diagram above with the corresponding diagram of cones [7, 2.4.13]:

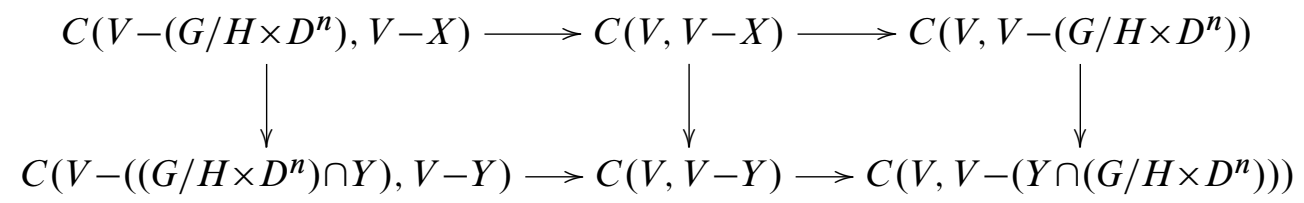

Now consider the set $U=\left(G / H \times D^{n}\right)-\left(\left(G / H \times D^{n}\right) \cap Y\right)$. The closure of $U$ in $V-\left(\left(G / H \times D^{n}\right) \cap Y\right)$ is contained in the interior of $V-Y$, and so excision [7, 2.4.3] implies that the leftmost map is a weak equivalence.

Therefore, upon application of $Z$ we obtain a homotopy pullback square:

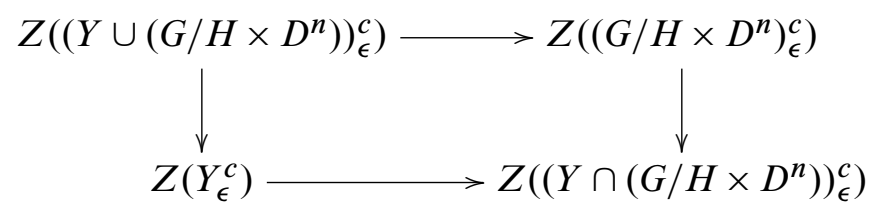

Algebraic $8 \mathcal{G}$ Geometric Topology, Volume 6 (2006) 
In addition, applying the mapping space functor $\operatorname{Map}_{0}\left(-, Z\left(S^{V}\right)\right)$ and recalling that it also takes cofibrations to fibrations, we have a homotopy pullback square

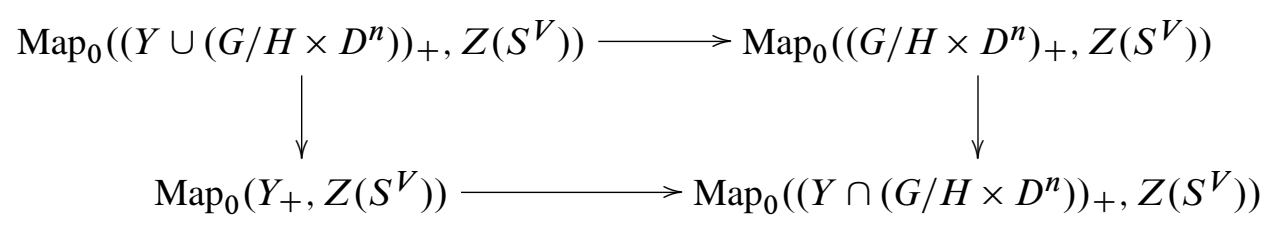

and one checks that the cube induced by the maps $\xi_{X}$ is commutative. Since $S(1)$ is regular, $Y \cap\left(G / H \times D^{n}\right)$ is a subcomplex consisting of strictly fewer cells (of lower dimension) and so the inductive hypothesis implies that we have equivalences at the three nonterminal corners of the cube, and therefore there is an equivalence at the terminal corner.

We now wish to show that in fact hypothesis (A) is necessary. In order to do so, we must first recall the following equivariant version of a theorem of Lydakis [8].

Proposition 3.6 Let $Z$ be a $\mathscr{W}_{G}$-space and $A \in \mathscr{W}_{G}$. Then the maps $Z_{U}(X) \wedge A \rightarrow$ $Z_{U}(X \wedge A)$ induce a $\pi_{*}$-equivalence $Z_{U}\left[S^{0}\right] \wedge A \simeq Z_{U}[A]$.

Proof The proof follows the nonequivariant proof given in $[10,17.6]$. In order to perform the induction, we substitute the equivariant theorems [9, 3.3.5] for [10, 7.4]. Note that we depend on the fact that all $A \in \mathscr{W}_{G}$ are stably dualizable when $U$ is a complete universe (as we are assuming here). See Section 3.4 for discussion of the situation when $U$ is not complete.

This result allows us to pass between $\pi_{*}$-equivalences of fibrant $\mathscr{W}_{G}$-spaces and weak equivalences of the "zero spaces" of the $\mathscr{W}_{G}$-spaces. We will employ this observation to deduce information about our space-level maps $\xi$ from stable dualities.

Notation 3.7 For a prespectrum $Z$, we will sometimes write $\Omega^{\infty} Z$ in place of $Z\left(S^{0}\right)$.

Corollary 3.8 Let $Y$ and $Z$ be $\mathscr{W}_{G}$-spaces which are fibrant in the absolute stable model structure. Denote the fibrant replacement of a prespectrum $D$ by $f D$.

(1) The spaces $\Omega^{\infty} f\left(Z_{U}\left[S^{0}\right] \wedge A\right)$ and $Z(A)$ are weakly equivalent.

(2) Given a map of prespectra $Y_{U}\left[S^{0}\right] \rightarrow Z_{U}\left[S^{0}\right]$ induced from a natural transformation $Y \rightarrow Z$ and a map $A \rightarrow B$ for $A, B \in \mathscr{W}_{G}$, if the induced map

$$
Y_{U}\left[S^{0}\right] \wedge A \rightarrow Z_{U}\left[S^{0}\right] \wedge B
$$


is a $\pi_{*}$-equivalence then the induced map

$$
Y(A) \rightarrow Z(B)
$$

is a weak equivalence.

(3) Given a map of prespectra $Y_{U}\left[S^{0}\right] \rightarrow Z_{U}\left[S^{0}\right]$ arising from a natural transformation $Y \rightarrow Z$ and a map $A \wedge B \rightarrow C$ for $A, B, C \in \mathscr{W}_{G}$, if the induced map

$$
Y_{U}\left[S^{0}\right] \wedge A \rightarrow F\left(B, Z_{U}\left[S^{0}\right] \wedge C\right)
$$

is a $\pi_{*}$-equivalence then the induced map

$$
Y(A) \rightarrow \operatorname{Map}_{0}(B, Z(C))
$$

is a weak equivalence.

Proof The first part is an immediate consequence of the preceding proposition. Take any $A \in \mathscr{W}_{G}$. Since there is a $\pi_{*}$-equivalence $Z_{U}\left[S^{0}\right] \wedge A \rightarrow Z[A]$, there is a $\pi_{*}$-equivalence $f\left(Z_{U}\left[S^{0}\right] \wedge A\right) \rightarrow f(Z[A])$. As these are $\Omega-G$-prespectra, a $\pi_{*}-$ equivalence is the same as a level equivalence [9, 3.3.4], and so there is a weak equivalence $\Omega^{\infty} f\left(Z_{U}\left[S^{0}\right] \wedge A\right) \rightarrow \Omega^{\infty} f(Z[A])$. But since there is also a level equivalence $Z[A] \rightarrow f(Z[A])$, the result follows.

For the second claim, the given maps induce a commutative diagram

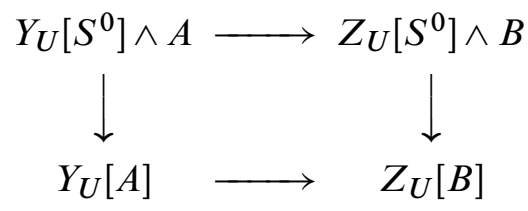

in which the vertical maps are $\pi_{*}$-equivalences. Therefore, if the top horizontal map is a $\pi_{*}$-equivalence, the bottom map must also be a $\pi_{*}$-equivalence. Since $Y_{U}[A]$ and $Z_{U}[B]$ are $\Omega-G$-prespectra by hypothesis, a $\pi_{*}$-equivalence is a level equivalence and therefore we have a weak equivalence $Y(A)=\Omega^{\infty} Y_{U}[A] \rightarrow \Omega^{\infty} Z_{U}[A]=Z(A)$. Using the naturality of the structure maps, we can see that this induced weak equivalence coincides with the map $Y(A) \rightarrow Z(B)$ induced from the natural transformation $Y \rightarrow Z$ and the map $A \rightarrow B$.

Finally, the last part follows from an argument similar to the second part. There is a commutative diagram

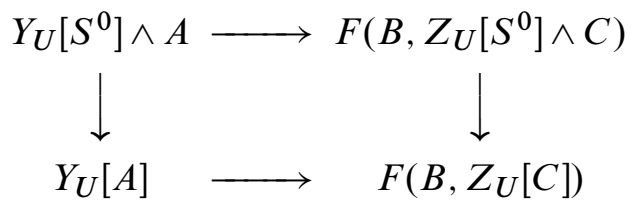

Algebraic $8 \mathcal{G}$ Geometric Topology, Volume 6 (2006) 
which arises as the adjoint of the commutative diagram

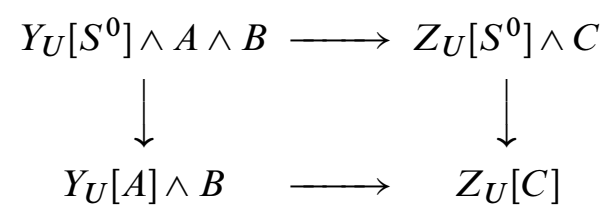

where the bottom vertical map is the composite of the structure map and the given map $A \wedge B \rightarrow C$. Now we argue as above, using the fact that for any space $B$ and prespectrum $Z, \Omega^{\infty} F(B, Z)=\operatorname{Map}_{0}\left(B, \Omega^{\infty} Z\right)$.

With this in hand, we can complete the proof that our condition on $\mathscr{W}_{G}$-spaces is necessary and sufficient for the prespectra $Z_{U}[A]$ to be $\Omega-G$-prespectra.

Theorem 3.9 For a $\mathscr{W}_{G}$-space $Z$, the following are equivalent.

(1) Z satisfies hypothesis (A).

(2) For any finite $G-C W$-complex $A, Z_{U}[A]$ forms an $\Omega-G$-prespectrum.

(3) For any finite $G-C W$-complex $A$, for any $W$ in the universe $U$, the adjoint of the structure map $Z(A) \wedge S^{W} \rightarrow Z\left(S^{W} \wedge A\right)$ is a weak equivalence

$$
Z(A) \simeq \Omega^{W} Z\left(\Sigma^{W} A\right) .
$$

Proof We have already shown that if $Z$ satisfies hypothesis $(A)$, then $Z_{U}[A]$ forms an $\Omega-G$-prespectrum for all $A \in \mathscr{W}_{G}$. By definition this is equivalent to the third condition. Now assume that $Z[A]$ is an $\Omega-G$-prespectrum for every $A \in \mathscr{W}_{G}$. The "naive" version of this result, Proposition 3.1, implies that $Z$ takes $G$-homotopy pushout squares to $G$-homotopy pullback squares.

Thus, we need to show that the map $\xi$ is an equivalence for all $G / H$ which embed in $V$ for any $V$ in the universe $U$. Let $E$ denote $Z_{U}\left[S^{0}\right]$, and recall this is an $\Omega-G$ prespectrum by hypothesis. By the third part of Corollary 3.8,

$$
\xi: Z(T \nu \wedge X) \rightarrow \operatorname{Map}_{0}\left(G / H_{+}, Z\left(S^{V} \wedge X\right)\right)
$$

will be a weak equivalence if the map

$$
\tilde{\xi}: E \wedge X \wedge(G / H)_{\epsilon}^{c} \rightarrow F\left(G / H_{+}, E \wedge S^{V} \wedge X\right)
$$

is a $\pi_{*}$-equivalence. Here $\tilde{\xi}$ is obtained by adjunction from the map

$$
E \wedge X \wedge(G / H)_{\epsilon}^{c} \wedge G / H_{+} \stackrel{\text { id } \wedge \text { id } \wedge \epsilon}{\longrightarrow} E \wedge X \wedge S^{V}
$$


One consequence of Spanier-Whitehead duality is that we can factor $\widetilde{\xi}$ as the composite

$$
E \wedge X \wedge(G / H)_{\epsilon}^{c} \rightarrow F\left(G / H_{+}, S^{V}\right) \wedge E \wedge X \rightarrow F\left(G / H_{+}, S^{V} \wedge E \wedge X\right) .
$$

Since $G / H_{+}$is dualizable, the second map is a $\pi_{*}$-equivalence. The first map is obtained from the duality equivalence

$$
\Sigma^{\infty}(G / H)_{\epsilon}^{c} \rightarrow F\left(G / H_{+}, S^{V}\right)
$$

by smashing with $E \wedge X$ on both sides.

Though $E \wedge X$ is not necessarily cofibrant, $\Sigma^{\infty}(G / H)_{\epsilon}^{c}$ is cofibrant and $F\left(G / H_{+}, S^{V}\right)$ is homotopy equivalent to a cofibrant $\mathscr{W}_{G}$-space, so the map is a $\pi_{*}$-equivalence.

Remark 3.10 One could also explicitly construct a homotopy inverse to the spacelevel map $Z(T \nu \wedge X) \rightarrow \operatorname{Map}_{0}\left(G / H_{+}, F\left(S^{V} \wedge X\right)\right)$ using $V$-duality. In our treatment, this is packaged up inside the machinery of Corollary 3.8 .

We will refer to $\mathscr{W}_{G}$-spaces satisfying these equivalent conditions as "genuinely equivariantly linear".

\subsection{Refinement via the Wirthmuller isomorphism}

Using the ideas that lead to the generalized Wirthmuller isomorphism, we can replace hypothesis (A) with a condition which does not explicitly involve $T v$. To do so, we must first digress and discuss the passage from $\mathscr{W}_{G}$-spaces to $\mathscr{W}_{H}$-spaces induced by an inclusion $H \rightarrow G$. There is a forgetful functor $\iota^{*}: \mathscr{T}_{G} \rightarrow \mathscr{T}_{H}$. Since $\iota^{*}(G / K)$ admits a triangulation as a finite $H-\mathrm{CW}-$ complex $[9,5.2 .2]$, this restricts to a forgetful functor $\iota^{*}: \mathscr{W}_{G} \rightarrow \mathscr{W}_{H}$.

Definition 3.11 Given $H \hookrightarrow G$, define $\iota^{*} Z$ as $\left(\iota^{*} Z\right)\left(\iota^{*} A\right)=\iota^{*}(Z(A))$.

Of course, not all $A \in \mathscr{W}_{H}$ are in the image of $\iota^{*}$, and thus what we have really produced is a continuous functor from $\iota^{*} \mathscr{W}_{G}$ to $H$-spaces. To obtain an $\mathscr{W}_{H}$-space, we apply the prolongation functor along the inclusion of $\iota^{*} \mathscr{W}_{G}$ in $\mathscr{W}_{H}$. This process is completely analogous to the construction of the change-of-group functors for classical prespectra, where restriction to indexing sequences and a change-of-universe are necessary. More precisely, $\iota^{*}$ on $\mathscr{W}_{G}$-spaces is compatible under the passage to prespectra with the usual change-of-group functor there, essentially by construction. That is, $\iota^{*} \mathbb{U} Z \cong \mathbb{U} \iota^{*} Z$.

Remark 3.12 One can show that there is a Quillen equivalence between the stable model structures on $\iota^{*} \mathscr{W}_{G}$-spaces and $\mathscr{W}_{H}$-spaces, by comparing each category to an appropriately indexed category of orthogonal spectra and using the observation that the change of universe functors are compatible. 
Henceforth, given a $\mathscr{W}_{G}$-space we will tacitly apply it to $H$-spaces and mean the corresponding $\mathscr{W}_{H}$-space produced in the fashion above. With this in hand, we proceed to revise hypothesis (A) using the Wirthmuller isomorphism.

The "neo-classical" construction of the Wirthmuller isomorphism given in [7] depends on a space-level $H$-map

$$
u: G \wedge_{H} X \rightarrow S^{L} \wedge X
$$

where $G / H$ is embedded in a representation $V, L$ is the associated tangent $H-$ representation at the identity, and $X$ is an arbitrary $H$-space. Applying $Z$ to both sides, we get an $H$-map

$$
Z\left(G \wedge_{H} X\right) \rightarrow Z\left(S^{L} \wedge X\right)
$$

Using the fact that $Z\left(G \wedge_{H} X\right)$ is regarded as an $H$-space by forgetting down from the $G$-space structure and the adjunction between the forgetful functor and $\mathrm{Map}_{H}$, we get an induced $G$-map

$$
\xi_{3}: Z\left(G \wedge_{H} X\right) \rightarrow \operatorname{Map}_{H}\left(G_{+}, Z\left(S^{L} \wedge X\right)\right) .
$$

Now, if we let $X$ be $S^{W}$ where $W \oplus L=V$ as an $H$-space, we get an $H$-map

$$
Z\left(G \wedge_{H} S^{W}\right) \rightarrow \operatorname{Map}_{H}\left(G_{+}, Z\left(S^{V}\right)\right)
$$

which corresponds to a $G$-map

$$
\xi_{3}: Z\left(G \wedge_{H} S^{W}\right) \rightarrow \operatorname{Map}_{0}\left(G / H_{+}, Z\left(S^{V}\right)\right) .
$$

Since $G \wedge_{H} S^{W}$ is precisely $T v$ in this setting, we can compare $\xi_{3}$ to $\xi_{2}$ (which was defined in Remark 3.2).

Lemma 3.13 Under the identification of $G \wedge_{H} S^{W}$ with $T v$, the maps $\xi_{3}$ and $\xi_{2}$ are $G$-homotopic.

Proof This is essentially a consequence of the observation [7, 2.5.9] that the Pontryagin-Thom map $S^{V} \rightarrow G \wedge_{H} S^{W}$ and the Wirthmuller map $u: G \wedge_{H} S^{W} \rightarrow S^{V}$ are compatible. That is, the composite

$$
S^{V} \rightarrow G \wedge_{H} S^{W} \rightarrow S^{V}
$$

is $H$-homotopic to the identity. By inspection, this permits the desired comparison of $\xi_{3}$ and $\xi_{2}$. 
Hypothesis 3.14 A $\mathscr{W}_{G}$-space $Z$ satisfies hypothesis (B) for the universe $U$ if the following two conditions hold.

(1) $Z$ takes $G$-homotopy pushout squares to $G$-homotopy pullback squares.

(2) Let $G / H$ embed in a representation $V$ in the universe $U$. Let $L$ be the tangent $H$-representation at the identity coset. Then for all $X \in \mathscr{W}_{H}$, the map

$$
\xi_{3}: Z\left(G \wedge_{H} X\right) \rightarrow \operatorname{Map}_{H}\left(G_{+}, Z\left(S^{L} \wedge X\right)\right)
$$

is a $G$-equivalence.

As one would hope, it turns out that this is equivalent to the previous condition. To prove this, we need a specialization of Corollary 3.8 .

Lemma 3.15 Let $Y$ and $Z$ be $W_{G}$-spaces such that for all $A \in \mathscr{W}_{G}$ the prespectra $Y_{U}[A]$ and $Z_{U}[A]$ are $\Omega-G$-prespectrum. Take $B, C \in \mathscr{W}_{H}$. Then given a map of prespectra $Y_{U}\left[S^{0}\right] \rightarrow Z_{U}\left[S^{0}\right]$ arising from a natural transformation $Y \rightarrow Z$ and a map of $H$-spaces $G \wedge_{H} B \rightarrow C \wedge B$, if the induced map of $G$-prespectra

$$
Y_{U}\left[S^{0}\right] \wedge\left(G \wedge_{H} B\right) \rightarrow F_{H}\left(G_{+}, Z_{U}\left[S^{0}\right] \wedge C \wedge B\right)
$$

is a $\pi_{*}$-equivalence then the induced map of $G$-spaces

$$
Y\left(G \wedge_{H} B\right) \rightarrow \operatorname{Map}_{H}\left(G_{+}, Z(C \wedge B)\right)
$$

is a weak equivalence.

Proof The argument is similar to the third part of Corollary 3.8. There is a commutative diagram of $G$-prespectra

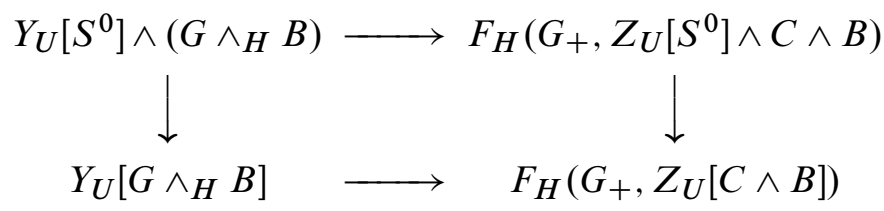

which arises as the adjoint of the commutative diagram of $H$-prespectra:

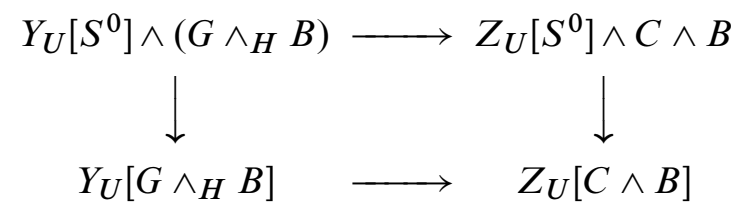

Now the result follows from the fact that for any $H$-prespectrum $X$,

$$
\Omega^{\infty} F_{H}\left(G_{+}, X\right)=\operatorname{Map}_{H}\left(G_{+}, \Omega^{\infty} X\right) .
$$


Theorem 3.16 A $\mathscr{W}_{G}$-space $Z$ satisfies hypothesis (A) if and only if it satisfies hypothesis (B).

Proof We will prove that hypothesis (B) implies hypothesis (A), and that if $Z_{U}[A]$ is an $\Omega-G$-spectrum for all $A \in \mathscr{W}_{G}$ then $Z$ satisfies hypothesis (B). The fact that hypothesis (B) implies hypothesis (A) is an immediate consequence of Lemma 3.13, which identifies $\xi_{3}$ with $\xi$.

On the other hand, if $Z_{U}[A]$ is an $\Omega-G$-prespectrum for all $A \in \mathscr{W}_{G}$ then hypothesis (B) holds as a consequence of the Wirthmuller isomorphism. Once again, let $E$ denote $Z_{U}\left[S^{0}\right]$. Using Lemma 3.15, the map

$$
\xi_{3}: Z\left(G \wedge_{H} X\right) \rightarrow \operatorname{Map}_{H}\left(G_{+}, Z\left(S^{L} \wedge X\right)\right)
$$

is a weak equivalence if the map

$$
\tilde{\xi_{3}}: E \wedge\left(G \wedge_{H} X\right) \rightarrow F_{H}\left(G_{+}, E \wedge\left(S^{L} \wedge X\right)\right)
$$

is a $\pi_{*}$-equivalence. The map $\tilde{\xi}_{3}$ is constructed as follows. Via application of $\Sigma^{\infty}, u$ induces a map of $H$-prespectra

$$
\mu: G \ltimes_{H} \Sigma^{\infty} X \rightarrow \Sigma^{L} \Sigma^{\infty} X
$$

and this induces a map of $G$-prespectra

$$
G \ltimes_{H} \Sigma^{\infty} X \rightarrow F_{H}\left(G_{+}, \Sigma^{L} \Sigma^{\infty} X\right)
$$

which is in fact the Wirthmuller map [7, 2.6.10]. Now smashing $\mu$ by $E$ (regarded as an $H$-prespectrum) on both sides prior to inducing to a map of $G$-prespectra yields a map

$$
G \ltimes_{H}(X \wedge E) \rightarrow F_{H}\left(G_{+}, \Sigma^{L}(E \wedge X)\right)
$$

and using the fact that $E$ is actually a $G$-prespectrum, this simplifies to

$$
\tilde{\xi}_{3}: E \wedge\left(G \wedge{ }_{H} X\right) \rightarrow F_{H}\left(G_{+}, E \wedge\left(S^{L} \wedge X\right)\right) .
$$

The Wirthmuller isomorphism tells us this is $\pi_{*}$-equivalence.

This provides the connection to the model-theoretic discussion.

Corollary 3.17 A $\mathscr{W}_{G}$-space $Z$ is fibrant in the absolute stable model structure if and only if $Z$ is genuinely equivariantly linear (satisfies the conditions of Theorem 3.9). 


\subsection{Incomplete universes}

In the previous sections (and in the proofs of the model structures given in the appendix), we assume that the universe $U$ is complete. This assumption enters into our arguments when we employ Spanier-Whitehead duality. In a complete universe, all finite $G-$ $\mathrm{CW}-$ complexes are stably dualizable. However, this is no longer true in incomplete universes; as we briefly mentioned in the introduction, the failure of Spanier-Whitehead duality in the trivial universe is one of the motivating factors for the use of $G$-spectra indexed on a complete universe.

Nonetheless, variants of our main results are valid when $U$ is not complete. Duality in incomplete universes has been carefully studied by Lewis [6]. For our purposes, an essential result of Lewis is that an orbit spectrum $\Sigma^{\infty}(G / H)_{+}$is dualizable in the stable category with respect to an incomplete universe $U$ if and only if $G / H$ embeds in $U$. Lewis has also carefully verified the existence of the Wirthmuller map we use in the context of incomplete universes. Therefore, we can obtain variants of hypotheses (A) and (B) which are valid in incomplete universes by restricting to orbits $G / H$ which embed in the universe.

The other essential modification is forced by the partial failure of the equivariant version of Lydakis' Proposition 3.6, which states that for a $W_{G}$-space $Z$ and any finite $G$-CW-complex $A$, there is a $\pi_{*}$-equivalence $Z_{U}\left[S^{0}\right] \wedge A \simeq Z_{U}[A]$. The proof of this proposition relies on $A$ being stably dualizable, and so when working over an incomplete universe we must restrict to $A \in \mathscr{W}_{G}$ which are dualizable. We employ this proposition to prove both the existence of the absolute stable model structure (and the associated characterization of fibrant objects) as well as to show the sufficiency of our hypotheses. Therefore, when working over an incomplete universe we need to restrict the quantification to stably dualizable $A$.

\subsection{Remarks on equivariant infinite loop space theory}

These fibrancy criteria provide a conceptual understanding of the marked difference between equivariant infinite loop space theory for $G$ finite and for $G$ an infinite compact Lie group. To be precise, we will first review the $\Gamma$-space approach to equivariant infinite loop space theory for a finite group $G$. There are two obvious approaches to generalizing the nonequivariant theory of $\Gamma$-spaces. A direct generalization is to consider $\Gamma-G$-spaces, which are functors from finite pointed sets to $G$-spaces. Alternatively, one could consider $\Gamma_{G}$-spaces, continuous functors from the category of finite pointed $G$-sets to $G$-spaces. It turns out that these categories are equivalent. This comparison was observed by Shimakawa [18], and is an example of a general fact about 
diagram spectra which (in the context of orthogonal $G$-spectra) is comprehensively discussed as part of the treatment of change-of-universe functors in $[9,5.1]$.

A $\Gamma_{G}$-space $X$ is "special" if the natural map

$$
X(G / H) \rightarrow \operatorname{Map}_{0}\left(G / H_{+}, X(1)\right)
$$

is an equivariant weak equivalence. This condition is equivalent to the usual condition on $\Gamma-G$-spaces [18]. Associated to a $\Gamma_{G}$-space $X$ via prolongation is a $\mathscr{W}_{G}$-space $\mathbb{P} X$. The main theorem in this setting is that a "very special" $\Gamma_{G}$-space gives rise to an $\mathscr{W}_{G}$-space which almost satisfies our hypothesis (A). Specifically, it only takes some homotopy pushouts squares to homotopy pushout squares. However, it turns out that enough of hypothesis (A) is satisfied for the prespectrum $\mathbb{U P} X$ to be identifiable as a positive $\Omega-G$-prespectra. Recall that a positive $\Omega-G$-prespectrum is a $G-$ prespectrum $Y$ such that the adjoint structure maps $Y(V) \rightarrow \Omega^{W} Y(V \oplus W)$ are weak equivalences for $W$ such that $W^{G} \neq 0$.

Remark 3.18 Analogous to the positive stable model structure on orthogonal $G-$ spectra there is a positive stable model structure on $\mathscr{W}_{G}$-spaces, obtained using identical arguments to those presented above to construct the stable model structure.

Consider hypothesis (B) in the case when $G$ is finite. Then we know that $\Sigma^{\infty} G / H_{+}$ is self-dual or equivalently $\Sigma^{\infty} T v$ is the same as $\Sigma^{\infty} G / H_{+}$. Therefore, hypothesis (B) amounts to requiring that the map

$$
Z\left(G \wedge_{H} X\right) \rightarrow \operatorname{Map}_{H}\left(G / H_{+}, Z(X)\right)
$$

be a $G$-equivalence. Plugging in $S^{0}$, we recover the "special" condition on the underlying $\Gamma_{G}$-space.

Now let $G$ be an infinite compact Lie group. In this setting, we can only consider $\Gamma-G$-spaces, as finite sets do not admit interesting $G$-actions. The dual of $\Sigma^{\infty} G / H_{+}$ is $G \ltimes S^{-L}$, and the representation sphere $S^{-L}$ is often nontrivial. Even restricting to $X=S^{0}$ in hypothesis (B), we must consider the map

$$
Z(G / H) \rightarrow \operatorname{Map}_{H}\left(G_{+}, Z\left(S^{L}\right)\right) .
$$

It is difficult to imagine how this equivalence could be encoded by entirely discrete data.

Instead, these requirements strongly suggests that a reasonable domain category for the correct analogue of $\Gamma$-spaces must contain enough information to encode these dualities, and therefore most likely should contain the orbit spectra $G / H$. Finally, it is worth pointing out that in general when $H$ has finite index in $G$, then $L$ is also trivial. 
Amongst other things, this suggests that infinite loop space theory for profinite groups when restricting the universe to finite index subgroups should be tractable.

\section{Appendix A Model category structures on $G \mathscr{W} \mathscr{T}$}

In this section we will analyze $\mathscr{W}_{G}$-spaces as equivariant diagram spectra. Closely following [9], we will construct stable model structures on $G \mathscr{W} \mathscr{T}$, compare these model structures to the stable model structure on orthogonal $G$-spectra, and provide a model-theoretic characterization of the fibrant objects.

\section{A.1 A rapid review of $G$-topological model categories}

The model structures we construct on $G \mathscr{W} \mathscr{T}$ are compatible with the enrichment in based $G$-spaces. This is expressed by an appropriate variant of Quillen's SM7 axiom, as follows. We briefly recall from $[9,3.1 .4]$ the following definition. Assume that we have a $G$-category $\mathscr{C}_{G}$, and its associated category of $G$-maps $G \mathscr{C}$. Let $i: A \rightarrow X$ and $p: E \rightarrow B$ be maps in $G \mathscr{C}$ and consider the map

$$
\mathscr{C}_{G}\left(i^{*}, p_{*}\right): \mathscr{C}_{G}(X, E) \rightarrow \mathscr{C}_{G}(A, E) \times_{\mathscr{C}_{G}(A, B)} \mathscr{C}_{G}(X, B)
$$

induced by $\mathscr{C}_{G}(i$,id $)$ and $\mathscr{C}_{G}($ id, $p)$ by passage to pullbacks.

Definition A.1 A model category is $G$-topological if the map $\mathscr{C}_{G}\left(i^{*}, p_{*}\right)$ is a Serre fibration (of $G$-spaces) when $i$ is a cofibration and $p$ is a fibration and is a weak equivalence when in addition either map is a weak equivalence.

\section{A.2 The stable model structure}

The construction of the various model structures on $G \mathscr{W} \mathscr{T}$ is mostly formal, using the technology developed in [9] and [10]. In the remainder of this section the predominant emphasis is on recording results along with carefully verifying the specific variant technical lemmas necessary for this situation. The interested reader should refer to the cited sources to reconstruct full arguments.

Remark A.2 Note that we will provide model structures only for $G \mathscr{W} \mathscr{T}$; it isn't very useful to talk about such structure on $\mathscr{W}_{G} \mathscr{T}$. Nonetheless, $\mathscr{W}_{G} \mathscr{T}$ is an important device for encoding the compatibility of the model structure on $G \mathscr{W} \mathscr{T}$ with the enrichment.

Throughout, fix a complete universe $U$. The first model structure we consider is the relative level model structure on $G \mathscr{W} \mathscr{T}$, where by relative we mean that the fibrations and weak equivalences are detected only on the spheres $\left\{S^{V}\right\}$ for $V \in U$. 
Definition A.3 The relative level model structure on this category is defined as follows. A map $Y \rightarrow Z$ is

(1) a fibration if each $Y\left(S^{V}\right) \rightarrow Z\left(S^{V}\right)$ is an equivariant Serre fibration,

(2) a weak equivalence if each $Y\left(S^{V}\right) \rightarrow Z\left(S^{V}\right)$ is an equivariant weak equivalence,

(3) a cofibration if it has the left-lifting property with respect to the acyclic fibrations.

Proposition A.4 The relative level model structure on $G \mathscr{W} \mathscr{T}$ is a cofibrantly generated $G$-topological model structure.

Proof The arguments are the same as [9, 3.2.4].

There is an associated stable model structure. We define $\pi_{*} Z$ for a $\mathscr{W}_{G}-$ space $Z$ by passing to the $G$-prespectrum $\mathbb{U} Z$ and specifying $\pi_{*} Z=\pi_{*} \cup Z$. Recall that for a subgroup $H$ of $G$ and an integer $q$, for $q \geq 0$ we define

$$
\pi_{q}^{H}(\mathbb{U} Z)=\operatorname{colim}_{V} \pi_{q}^{H}\left(\Omega^{V} Z(V)\right)
$$

and for $q>0$ we define

$$
\pi_{-q}^{H}(\mathbb{U} Z)=\operatorname{colim}_{V \supseteq \mathbb{R}^{q}} \pi_{0}^{H}\left(\Omega^{V-\mathbb{R}^{q}} Z(V)\right) .
$$

Definition A.5 In the stable model structure, a map is

(1) a cofibration if it is a cofibration in the relative level model structure,

(2) a weak equivalence if it is a $\pi_{*}$-equivalence,

(3) a fibration if it has the right-lifting property with respect to the acyclic cofibrations (maps which are both level cofibrations and $\pi_{*}$-equivalences).

We can employ the argument of $[9,3.4 .2]$ to prove that this is a model structure, but we need to specialize a lemma to the current situation. Recall that $A \mapsto F_{B} A$ as a functor from $G$-spaces to $\mathscr{W}_{G}$-spaces is defined to be left adjoint to the functor which is evaluation at $B$. Concretely, we have $\left(F_{B} A\right)(C)=\operatorname{Map}_{0}(B, C) \wedge A$.

There is a map

$$
\lambda_{V, A}: F_{\Sigma^{V} A} S^{V} \rightarrow F_{A} S^{0}
$$

defined to be map such that

$$
\lambda_{V, A}^{*}: \mathscr{W}_{G} \mathscr{T}\left(F_{A} S^{0}, X\right) \rightarrow \mathscr{W}_{G} \mathscr{T}\left(F_{\Sigma^{V}} S^{V}, X\right)
$$

corresponds under adjunction to

$$
X(A) \rightarrow \Omega^{V} X\left(\Sigma^{V} A\right) .
$$

The functors $\lambda_{V, A}$ play a key role in constructing the stable model structures, as they allow us to provide explicit descriptions of the generating cofibrations. 
Lemma A.6 For all based $G-C W$-complexes $B$, the maps

$$
\lambda_{V, A} \wedge \mathrm{id}: F_{\Sigma^{V} A}\left(\Sigma^{V} B\right) \cong F_{\Sigma^{V} A} S^{V} \wedge B \rightarrow F_{A} S^{0} \wedge B \cong F_{A} B
$$

are $\pi_{*}$-equivalences.

Proof We can write the specified map at a sphere $S^{Z}$ as

$$
\operatorname{Map}_{0}\left(S^{V} \wedge A, S^{Z}\right) \wedge S^{V} \wedge B \rightarrow \operatorname{Map}_{0}\left(A, S^{Z}\right) \wedge B .
$$

Rewriting, this is

$$
\left(\Sigma^{V} \Omega^{V} \operatorname{Map}_{0}\left(A, S^{Z}\right)\right) \wedge B \rightarrow \operatorname{Map}_{0}\left(A, S^{Z}\right) \wedge B
$$

and the map is the evaluation map. First, we can assume that $B=S^{0}$. It will suffice to show that the map is a $\pi_{*}$-equivalence in this case, since $B$ is a $G-\mathrm{CW}-$ complex and hence smashing with $B$ preserves $\pi_{*}$-equivalences. But observe that $\operatorname{Map}_{0}\left(A, S^{Z}\right)$ is describing the $Z$-th space of the cotensor prespectrum $F(A, S)$, and the map in question is a stable equivalence because the unit $\Sigma^{V} \Omega^{V} X \rightarrow X$ is a stable equivalence of prespectra.

Remark A.7 Note that we could also prove this directly by induction over cell decompositions, as is done in $[10,17.1]$, if a self-contained proof was desired that did not require the prior work on prespectra.

We need the following corollary, which trivially follows by setting $A=S^{W}$ in the lemma.

Corollary A.8 For all based $G-C W$ complexes $B$, the maps

$$
\lambda_{V, S^{W}} \wedge \text { id: } F_{S^{V \oplus W}} \Sigma^{V} B \cong F_{S^{V \oplus W}} S^{V} \wedge B \rightarrow F_{S^{W}} S^{0} \wedge B \cong F_{S^{W}} B
$$

are $\pi_{*}$-isomorphisms.

We define the generating cofibrations as follows. First, recall the sets $I$ and $J$ from [9, 3.1.1]; $I$ is the set of cell cofibrations

$$
i:\left(G / H \times S^{n-1}\right)_{+} \rightarrow\left(G / H \times D^{n}\right)_{+}
$$

and $J$ is the set of cofibrations

$$
i_{0}:\left(G / H \times D_{n}\right)_{+} \rightarrow\left(G / H \times D^{n} \times[0,1]\right)_{+} .
$$

Here $H$ runs through the closed subgroups of $G$ and $n \geq 0$. 
Definition A.9 The set $F I$ is the set of all maps $F_{S^{V}} i$ for $i \in I$ and $V \subset U$. The set $F J$ is the set of all maps $F_{S^{V}} j$ for $j \in J$ and $V \subset U$.

We need to define the operation $f \square g$ for maps $f$ and $g$ in order to specify the generating acyclic cofibrations. If $i: X \rightarrow Y$ and $j: W \rightarrow Z$ are cofibrations, then there is a cofibration

$$
i \square j:(Y \wedge W) \cup_{X \wedge W}(X \wedge Z) \rightarrow Y \wedge Z .
$$

Definition A.10 Let $M \lambda_{V, S^{W}}$ be the mapping cylinder of $\lambda_{V, S^{W}}$. Then $\lambda_{V, S^{W}}$ factors as the composite of a cofibration $k_{V, W}: F_{S^{V \oplus W}} S^{W} \rightarrow M \lambda_{V, S^{W}}$ and a deformation retraction $r_{V, W}: M \lambda_{V, S} \rightarrow F_{S^{V}} S^{0}$. Let $K$ be the union of $F J$ and maps of the form $i \square k_{V, W}, i \in I$.

Using Corollary A.8, the arguments of $[9,3.4 .2]$ then imply the following result.

Proposition A.11 The stable model structure is a $G$-topological model structure on the category $G \mathscr{W} \mathscr{T}$ with generating cofibrations $F I$ and generating acyclic cofibrations $K$.

\section{A.3 Comparison to orthogonal $G-$ spectra}

There is a pair of adjoint functors $(\mathbb{P}, \mathbb{U})$ connecting $G \mathscr{W} \mathscr{T}$ and the category $G \mathscr{I S}$ of orthogonal $G$-spectra $[9 ; 10] . \mathbb{U}$ is the forgetful functor from $\mathscr{W}_{G}$-spaces to orthogonal $G$-spectra, and $\mathbb{P}$ is the prolongation constructed as a left Kan extension along the inclusion of domain categories [10, 23.1]. As an immediate consequence of [9, 2.14], we find that these functors preserve the symmetric monoidal structures.

Lemma A.12 $\mathbb{P}$ is a strong symmetric monoidal functor and $\mathbb{U}$ is a lax symmetric monoidal functor.

Moreover, we have the expected comparison result.

Theorem A.13 The pair $(\mathbb{P}, \mathbb{U})$ specifies a Quillen equivalence between the stable model category structure on $G \mathscr{W} \mathscr{T}$ and the stable model category structure on $G \mathscr{I} \mathscr{S}$.

Proof This is virtually identical to the comparison between orthogonal $G$-spectra and $G$-prespectra $[9,4.16]$. 


\section{A.4 The absolute stable model structure}

The level model structure used to construct the stable model structure in the previous section depends on evaluation at the spheres. This makes it inconvenient to compare to diagram categories where the domain does not include an embedding of the spheres, for instance $\Gamma-G$-spaces. As in the nonequivariant case, we rectify this by considering an "absolute" model structure.

Definition A.14 The absolute level model structure on $G \mathscr{W} \mathscr{T}$ is defined as follows. A map $Y \rightarrow Z$ is

(1) a fibration if each $Y(A) \rightarrow Z(A)$ for $A \in \mathscr{W}_{G}$ is an equivariant Serre fibration,

(2) a weak equivalence if each $Y(A) \rightarrow Z(A)$ for $A \in \mathscr{W}_{G}$ is an equivariant weak equivalence,

(3) a cofibration if it has the left-lifting property with respect to the acyclic fibrations.

Proposition A.15 The absolute level model structure on $G \mathscr{W} \mathscr{T}$ is a cofibrantly generated $G$-topological model structure.

Proof Again, the arguments are the same as [9, 3.2.4].

There is an associated absolute stable model structure.

Definition A.16 In the absolute stable model structure, a map is

(1) a cofibration if it is a cofibration in the absolute level model structure,

(2) a weak equivalence if it is a $\pi_{*}$-equivalence,

(3) a fibration if it has the right-lifting property with respect to the acyclic cofibrations.

In order to prove that these definitions yield a model structure on the category of $\mathscr{W}_{G^{-}}$ spaces, we require the full strength of Lemma A.6, which tells us that the maps $\lambda_{V, A}$ are $\pi_{*}$-equivalences. To obtain the generating cofibrations and acyclic cofibrations, we enlarge $F I$ and $F J$ by defining $F^{\prime} I$ to be the set of all maps $F_{A} i$ for $i \in I$ and $A \in \mathscr{W}_{G}$ and $F^{\prime} J$ to be the set of all maps $F_{A} j$ for $j \in J$. We then construct $K^{\prime}$ analogously to $K$, taking mapping cylinders for all maps $\lambda_{V, A}$.

Proposition A.17 The stable model structure is a $G$-topological model structure on the category $G \mathscr{W} \mathscr{T}$ with generating cofibrations $F^{\prime} I$ and generating acyclic cofibrations $K^{\prime}$. 
Proof This follows the proof of [10, 17.2], modified slightly in light of the proof of $[9,3.4 .2]$.

In the course of this proof we obtain the following analogue of $[9,3.4 .8]$.

Proposition A.18 A $\mathscr{W}_{G}$-space $Z$ is fibrant in the absolute stable model structure if and only if for all $A \in \mathscr{W}_{G}$ and $W \in U$ the structure map

$$
Z(A) \rightarrow \Omega^{W} Z\left(S^{W} \wedge A\right)
$$

is a weak equivalence.

Finally, we can compare the absolute and relative stable model structures. It is clear that the identity functor is the left adjoint in a Quillen pair relating the two model structures.

Theorem A.19 The identify functor is the left adjoint of a Quillen equivalence between the category of $\mathscr{W}_{G}$-spaces with the relative stable model structure and the category of $\mathscr{W}_{G}$-spaces with the absolute stable model structure.

\section{A.5 Ring and module spectra}

We can leverage the results of $[9 ; 10]$ to lift the stable model structure to categories of ring and module $\mathscr{W}_{G}$-spaces. The key ingredient in these lifting results is the verification of the monoid axiom and the pushout-product axiom.

To verify these, we need the following technical lemma.

Lemma A.20 Let $Y$ be a $\mathscr{W}_{G}$-space such that $\pi_{*}(Y)=0$. Then $\pi_{*}\left(F_{V} S^{V} \wedge Y\right)=0$ for any $V$.

Proof The conclusion follows immediately from the counterpart for orthogonal $G-$ spectra $[9,3.7 .2]$ upon applying the prolongation functor $\mathbb{P}$ to $\mathscr{W}_{G}$-spaces, just as in $[10,12.3]$.

Now, the same chain of arguments given in $[9,3.7]$ allows us to verify the following two results.

Proposition A.21 (Monoid axiom) For any acyclic cofibration $i: X \rightarrow Y$ of $\mathscr{W}_{G^{-}}$ spaces and any $\mathscr{W}_{G}$-space $Z$, the map $i \wedge$ id: $X \wedge Z \rightarrow Y \wedge Z$ is a $\pi_{*}$-isomorphism and an $h$-cofibration. This holds for cobase changes and sequential colimits of such maps as well.

Algebraic $8 \mathcal{G}$ Geometric Topology, Volume 6 (2006) 
Note that by $h$-cofibration we mean a map satisfying the homotopy extension property as opposed to a model theoretic cofibration.

Proposition A.22 (Pushout-product axiom) If $i: X \rightarrow Y$ and $j: W \rightarrow Z$ are cofibrations of $\mathscr{W}_{G}$-spaces and $i$ is a $\pi_{*}$-isomorphism, then the cofibration

$$
i \square j:(Y \wedge W) \cup_{X \wedge W}(X \wedge Z) \rightarrow Y \wedge Z
$$

is a $\pi_{*}$-isomorphism.

As an immediate consequence, we have the following version of $[9,3.7 .6]$.

Theorem A.23 Let $R$ be a ring $\mathscr{W}_{G}$-space.

(1) The category of $R$-module $\mathscr{W}_{G}$-spaces is a cofibrantly generated proper $G$ topological model category, with weak equivalences and fibrations created in the stable model structure on the category of $\mathscr{W}_{G}$-spaces.

(2) If $R$ is commutative, the category of $R$-algebra $\mathscr{W}_{G}$-spaces is a cofibrantly generated right proper $G$-topological model category with weak equivalences and fibrations created in the stable model structure on the category of $\mathscr{W}_{G}$-spaces.

Remark A.24 Following the outline above, one can also obtain a version of this theorem by lifting the absolute stable model structure. This variant of the theorem requires a slightly stronger version of Lemma A.20, obtained by an equivariant version of the argument for $[10,17.6]$.

\section{Appendix B The failure of the approximation theorem for $G=S^{1}$}

An equivariant approximation theorem would purport to show that an appropriate map $C(V) \rightarrow \Omega^{V} S^{V}$ was a group completion, where $C(V)$ is the configuration space of points of $V$. Note that this formulation would actually be correct only for $V$ such that $V^{G}$ is nonzero. The statement is somewhat more complicated when $V^{G}=0$, as then there is no addition.

This counterexample is due to Segal [16]. Let $G=S^{1}$ and let $V=\mathbb{R}^{3}$ where $G$ acts by rotation around the $z$-axis. The inclusion of the axis gives a cofibration $S^{1} \rightarrow S^{V}$ of $G$-spaces, and the cofiber $S^{V} / S^{1}$ is $G$-homeomorphic to $\Sigma^{2} G_{+}$. To see this, let $G$ be the unit circle in the $(x, y)$-plane with the disjoint basepoint at the origin, and parameterize $\mathbb{R}^{3}$ as $S^{1} \times[0, \infty) \times \mathbb{R} /(t, 0, s) \sim\left(t^{\prime}, 0, s\right)$. 
Choose a particular $G$-space $X$. There results a $G$-fibration sequence

$$
\operatorname{Map}_{0}\left(\Sigma^{2} G_{+}, X\right) \rightarrow \operatorname{Map}_{0}\left(S^{V}, X\right) \rightarrow \operatorname{Map}_{0}\left(S^{1}, X\right) .
$$

Passing to fixed-points, this remains a fibration sequence. Evaluating the fixed-points of the terms in the sequence, we find

$$
\operatorname{Map}_{0}\left(S^{1}, X\right)^{G} \simeq \Omega\left(X^{G}\right)
$$

and

$$
\operatorname{Map}_{0}\left(\Sigma^{2} G_{+}, X\right)^{G} \cong \operatorname{Map}_{0}\left(G_{+}, \Omega^{2} X\right)^{G} \cong \Omega^{2} X .
$$

Thus, we have the fibration sequence

$$
\Omega^{2} X \rightarrow\left(\Omega^{V} X\right)^{G} \rightarrow \Omega\left(X^{G}\right) .
$$

Finally, take $X=S^{V}$. Then $X^{G}=S^{1}$ and $\Omega S^{1} \simeq \mathbb{Z}$. The sequence splits and so we have $\left(\Omega^{V} S^{V}\right)^{G} \simeq \mathbb{Z} \times \Omega^{2} S^{3}$. But $G$-fixed points of the configuration space $C(V)^{G}$ are the same as the configuration space $C\left(\mathbb{R}^{1}\right)$, and the usual group completion theorem tells us that the group completion of this is $\Omega S^{1}$. Therefore, we have a contradiction.

\section{Appendix C The trouble with $\Gamma-S^{1}$-spaces}

In naive analogy with the situation for $G$ finite, one might hope that there is some condition on a $\Gamma-S^{1}$-space $F$ which would guarantee that the $W_{S^{1}}$-space $\mathbb{P} F$ obtained by prolongation would be a positive $\Omega-S^{1}$-spectrum [16; 17]. However, we will show that no such condition can exist by studying the counit of the $(\mathbb{P}, \mathbb{U})$ adjunction.

Any satisfactory condition would certainly be satisfied by a $\Gamma-S^{1}$-space obtained by forgetting from a genuinely equivariantly linear $W_{S^{1}}$-space. Consider the case in which we begin with a fibrant $W_{S^{1}}$-space $X$ such that the prespectrum $\mathbb{U} X$ is connective. Let $Y$ be the $\Gamma-S^{1}$-space obtained from $X$ via the forgetful functor, and denote by $\tilde{X}$ the prolongation $\mathbb{P} Y$. The counit of the adjunction gives us a map $\tilde{X} \rightarrow X$. We will compare $\tilde{X}$ and $X$, and show that in fact they will almost never be stably equivalent. As a consequence, there can be no condition on a $\Gamma-S^{1}$-space which will guarantee that its prolongation is a positive $\Omega-S^{1}$-spectrum.

We will proceed by comparing the associated $G$-prespectra $\mathbb{U} \tilde{X}$ and $\mathbb{U} X$. Abusing notation, we will also refer to these $G$-prespectra as $\tilde{X}$ and $X$. First, let $H \subset S^{1}$ be a finite subgroup. $Y$ determines a $\Gamma-H$-space $Y_{H}$ via the forgetful functor, and there is an associated $\Gamma_{H}$-space which we will also denote $Y_{H}$. Now, know that $X$ satisfies hypothesis (B) and therefore $Y_{H}$ is very special. Therefore, $\mathbb{P} Y_{H}$ is fibrant and there is an equivalence $\tilde{X}\left(S^{V}\right)^{H} \simeq X\left(S^{V}\right)^{H}$. 
Remark C.1 The previous observation is the starting point for a comparison of cyclic $\Gamma$-spaces and the $\mathscr{F}$-model structure on connective $S^{1}$-spectra. Here the weak equivalences on $S^{1}$-spectra are taken to be the $\mathscr{F}$-equivalences $[9,4.6 .5]$, where $\mathscr{F}$ denotes the family of finite subgroups of $S^{1}$. We intend to discuss this comparison elsewhere.

Now consider the $S^{1}$ fixed-points of $\tilde{X}\left(S^{V}\right)$.

Lemma C.2 There is a weak equivalence $\tilde{X}\left(S^{V}\right) S^{1} \simeq X\left(S^{V^{1}}\right) S^{1}$.

Proof By definition, $\tilde{X}\left(S^{V}\right)$ is the coend

$$
\int_{\Gamma} X(n) \times\left(S^{V}\right)^{n}
$$

Since $S^{1}$ is infinite, observe that $\tilde{X}\left(S^{V}\right)^{S^{1}}$ is in fact the same as

$$
\int_{\Gamma} X(n)^{S^{1}} \times\left(S^{V^{S^{1}}}\right)^{n}
$$

This implies that $\tilde{X}\left(S^{V}\right)^{S^{1}} \simeq X\left(S^{V^{S^{1}}}\right)^{S^{1}}$.

As an consequence, observe that there is a levelwise weak equivalence of prespectra $\tilde{X}^{S^{1}} \rightarrow X^{S^{1}}$. This observation allows us to obtain a precise description of the $G-$ prespectrum $\tilde{X}$. Recall that $E \mathscr{F}$ is the classifying space of the family of finite subgroups of $S^{1}$, so that $\left(E \mathscr{F}_{+}\right)^{H} \simeq S^{0}$ and $\left(E \mathscr{F}_{+}\right)^{S^{1}}$ contractible.

Proposition C.3 There is a zig-zag of levelwise weak equivalences of $G$-prespectra between $\tilde{X}$ and the following homotopy pushout:

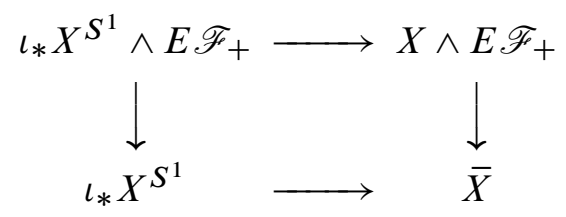

Proof Since $\iota_{*} X^{S^{1}}(V) \cong X\left(V^{S^{1}}\right) \wedge S^{V-V^{S^{1}}}$, the structure maps of $X$ induce the map in the top righthand corner. To compute the homotopy pushout, since the level model structure on $\mathscr{P}$ is left proper we can take the actual pushout in the diagram obtained by replacing $\iota_{*} X^{S^{1}} \wedge E \mathscr{F}+$ by a cofibrant $G$-prespectrum and the map $\iota_{*} X^{S^{1}} \wedge E \mathscr{F}_{+} \rightarrow X \wedge E \mathscr{F}+$ by a cofibration. The fixed-point functor commutes with this pushout, since one leg of the diagram is a cofibration and hence a closed 
inclusion [9, 3.1.6]. When we apply $(-)^{S^{1}}$, the top row is contractible and so there is an equivalence $\left(\iota_{*} X^{S^{1}}\right)^{S^{1}} \simeq \bar{X}^{S^{1}}$. When we apply $(-)^{H}$ for $H$ a finite subgroup of $S^{1}$, the left column becomes an equivalence and so we have $X^{H} \simeq \bar{X}^{H}$. To obtain the connection between $\tilde{X}$ to $\bar{X}$, we consider the analogous homotopy pushout:

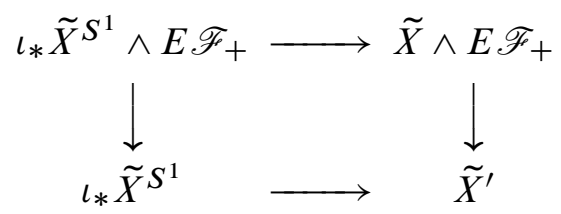

The map $\tilde{X} \rightarrow X$ induces a map of homotopy pushouts, which is a weak equivalence at each corner by previous discussion. Therefore there is an equivalence $\tilde{X}^{\prime} \rightarrow \bar{X}$. There is also a map from $\tilde{X}^{\prime} \rightarrow \tilde{X}$ obtained from the natural maps $\iota_{*} \tilde{X}^{S^{1}} \rightarrow \tilde{X}$ and $\tilde{X} \wedge E \mathscr{F}_{+} \rightarrow \tilde{X}$. This map clearly becomes an equivalence upon application of $(-)^{H}$ for $H \subset S^{1}$ finite. Applying $(-)^{S^{1}}$ to the associated pushout diagram, the top row is contractible and on the bottom we obtain the composite

$$
\left(\iota_{*} \tilde{X}^{S^{1}}\right)^{S^{1}} \rightarrow\left(\tilde{X}^{\prime}\right)^{S^{1}} \rightarrow \tilde{X}^{S^{1}},
$$

which is a weak equivalence. Since $\left(\iota_{*} \tilde{X}^{S^{1}}\right) \rightarrow\left(\tilde{X}^{\prime}\right)^{S^{1}}$ is also a weak equivalence, the map $\left(\tilde{X}^{\prime}\right)^{S^{1}} \rightarrow \tilde{X}^{S^{1}}$ must be a weak equivalence.

Applying the spectrification functor $L$ we can use this description to compute the $S^{1}$-fixed points of $L \tilde{X}$. Recall that $L$ is the left adjoint in a Quillen equivalence between $\mathscr{P}$ with the stable model structure and $\mathscr{S}$ with the generalized cellular model structure $[9,4.2 .9]$.

Lemma C.4 The spectrum $(L \tilde{X})^{S^{1}}$ is weakly equivalent to $\left(L\left(\iota_{*} X^{S^{1}}\right)\right)^{S^{1}}$.

Proof The pushout square describing $\bar{X}$ as a $G$-prespectrum is taken to a pushout of $G$-spectra by $L$. For this calculation, it is convenient to assume that we have replaced both maps in the original square by cofibrations when computing $\bar{X}$. Then since the maps in the pushout are levelwise cofibrations and hence stable cofibrations, $L$ takes them to cofibrations of $G$-spectra. This implies that the resulting square of $G$-spectra is in fact a homotopy pushout. Therefore it is also a homotopy pullback square. The homotopy pullback can be computed by taking the actual pullback in the square

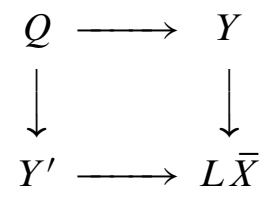

Algebraic 83 Geometric Topology, Volume 6 (2006) 
where $Y \rightarrow L \bar{X}$ and $Y^{\prime} \rightarrow L \bar{X}$ are fibrations, $Y \simeq L(X \wedge E \mathscr{F}), Y^{\prime} \simeq L\left(\iota_{*} X^{S^{1}}\right)$, and $Q \simeq L\left(\left(\iota_{*} X\right)^{S^{1}} \wedge E \mathscr{F}\right)$. Applying $(-)^{S^{1}}$, we observe that $Q^{S^{1}}$ and $Y^{S^{1}}$ are contractible. Therefore, we obtain an equivalence $\left(L\left(\iota_{*} X^{S^{1}}\right)\right)^{S^{1}} \simeq(L \bar{X})^{S^{1}}$, and this implies the result.

However, it is rarely the case that such an equivalence holds for an arbitrary $S^{1}$ spectrum $X$. For instance, the equivariant tom-Dieck splitting [7, 5.11.1] tells us that such an equivalence does not hold for suspension spectra.

Remark C.5 In the unstable setting, it is possible to obtain a model category structure which captures equivariant $S^{1}$-homotopy theory by gluing together a simplicial set and a cyclic set [1]. The previous discussion can be interpreted as a demonstration that the stable analogue of this gluing argument fails. The natural approach would be to attempt to decompose an $S^{1}$-spectrum $X$ into the nonequivariant $S^{1}$-fixed points $X^{S^{1}}$ and the "cyclic" part $X \wedge E \mathscr{F}_{+}$. To recover $X$, one would then glue $X \wedge E \mathscr{F}_{+}$ to the $S^{1}$-spectrum induced from $X^{S^{1}}, \iota_{*} X^{S^{1}}$. But the argument above shows that we cannot recapture the $S^{1}$-fixed points in this fashion. This is perhaps not surprising in light of the significant difference in complexity between the diagrams representing unstable equivariant spaces [4] and the diagrams representing stable equivariant objects [14].

\section{References}

[1] A J Blumberg, A discrete model of $\mathrm{S}^{1}$-homotopy theory, J. of Pure and Appl. Alg. (2006)

[2] J M Boardman, R M Vogt, Homotopy-everything $H$-spaces, Bull. Amer. Math. Soc. 74 (1968) 1117-1122 MR0236922

[3] S R Costenoble, $\mathbf{S}$ Waner, Fixed set systems of equivariant infinite loop spaces, Trans. Amer. Math. Soc. 326 (1991) 485-505 MR1012523

[4] A D Elmendorf, Systems of fixed point sets, Trans. Amer. Math. Soc. 277 (1983) 275-284 MR690052

[5] S Illman, The equivariant triangulation theorem for actions of compact Lie groups, Math. Ann. 262 (1983) 487-501 MR696520

[6] L G Lewis, Jr, Splitting theorems for certain equivariant spectra, Mem. Amer. Math. Soc. 144 (2000) MR1679450

[7] L G Lewis, Jr, JP May, M Steinberger (With contributions by J E McClure), Equivariant stable homotopy theory, Lecture Notes in Mathematics 1213, Springer, Berlin (1986) MR866482 
[8] M Lydakis, Simplicial functors and stable homotopy theory, preprint (1998)

[9] M A Mandell, J P May, Equivariant orthogonal spectra and $S$-modules, Mem. Amer. Math. Soc. 159 (2002) x+108 MR1922205

[10] M A Mandell, J P May, S Schwede, B Shipley, Model categories of diagram spectra, Proc. London Math. Soc. (3) 82 (2001) 441-512 MR1806878

[11] JP May, The geometry of iterated loop spaces, Lectures Notes in Mathematics 271, Springer, Berlin (1972) MR0420610

[12] JP May, Equivariant homotopy and cohomology theory, from: "Symposium on Algebraic Topology in honor of José Adem (Oaxtepec, 1981)", Contemp. Math. 12, Amer. Math. Soc., Providence, R.I. (1982) 209-217 MR676330

[13] J P May, The Wirthmüller isomorphism revisited, Theory Appl. Categ. 11 (2003) No. 5, 132-142 MR1988073

[14] S Schwede, B Shipley, Stable model categories are categories of modules, Topology 42 (2003) 103-153 MR1928647

[15] G Segal, Categories and cohomology theories, Topology 13 (1974) 293-312 MR0353298

[16] G Segal, Some results in equivariant homotopy theory, preprint (1975)

[17] K Shimakawa, Infinite loop $G$-spaces associated to monoidal $G$-graded categories, Publ. Res. Inst. Math. Sci. 25 (1989) 239-262 MR1003787

[18] K Shimakawa, A note on $\Gamma_{G}$-spaces, Osaka J. Math. 28 (1991) 223-228 MR1132161

Department of Mathematics, Stanford University

450 Serra Mall, Stanford, California 94305, USA

blumberg@math.stanford.edu

Received: 15 June 2005 Revised: 8 November 2006 